\title{
Controllability of the linear 1D wave equation with inner moving forces
}

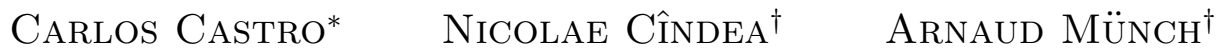

January 20, 2014

\begin{abstract}
This paper deals with the numerical computation of distributed null controls for the 1D wave equation. We consider supports of the controls that may vary with respect to the time variable. The goal is to compute approximations of such controls that drive the solution from a prescribed initial state to zero at a large enough controllability time. Assuming a geometric optic condition on the support of the controls, we first prove a generalized observability inequality for the homogeneous wave equation. We then introduce and prove the well-posedness of a mixed formulation that characterizes the controls of minimal square-integrable norm. Such mixed formulation, introduced in [Cindea and Münch, A mixed formulation for the direct approximation of the control of minimal $L^{2}$-norm for linear type wave equations], and solved in the framework of the (space-time) finite element method, is particularly well-adapted to address the case of time dependent support. Several numerical experiments are discussed.
\end{abstract}

Keywords: Linear wave equation, null controllability, finite element methods, Mixed formulation.

Mathematics Subject Classification (2010)- 35L10, 65M12, 93B40.

\section{Introduction}

Let $T$ be a positive real, $Q_{T}$ the domain $(0,1) \times(0, T), q_{T}$ a non-empty subset of $Q_{T}$ and $\Sigma_{T}=$ $\{0,1\} \times(0, T)$. We are concerned in this work with the null distributed controllability for the $1 \mathrm{D}$ wave equation:

$$
\begin{cases}y_{t t}-y_{x x}=v 1_{q_{T}}, & (x, t) \in Q_{T} \\ y(x, t)=0, & (x, t) \in \Sigma_{T} \\ y(x, 0)=y_{0}(x), \quad y_{t}(x, 0)=y_{1}(x), & x \in(0,1) .\end{cases}
$$

We assume that $\left(y_{0}, y_{1}\right) \in \boldsymbol{V}:=H_{0}^{1}(0,1) \times L^{2}(0,1) ; v=v(t)$ is the control (a function in $L^{2}\left(q_{T}\right)$ ) and $y=y(x, t)$ is the associated state. $1_{q_{T}}$ from $Q_{T}$ to $\{0,1\}$ denotes the indicatrice function of $q_{T}$. We also use the notation:

$$
L y:=y_{t t}-y_{x x} .
$$

For any $\left(y_{0}, y_{1}\right) \in \boldsymbol{V}$ and any $v \in L^{2}\left(q_{T}\right)$, there exists exactly one solution $y$ to (1), with the regularity $y \in C^{0}\left([0, T] ; H_{0}^{1}(0,1)\right) \cap C^{1}\left([0, T] ; L^{2}(0,1)\right)$ (see [19]).

\footnotetext{
*Dep. Matematica e Informatica, ETSI Caminos, Canales y Puertos, Universidad Politecnica de Madrid, 28040 Madrid, Spain. E-mail: carlos.castro@upm.es.

${ }^{\dagger}$ Laboratoire de Mathématiques, Université Blaise Pascal (Clermont-Ferand 2), UMR CNRS 6620, Campus de Cézeaux, 63177, Aubière, France. E-mails: nicolae.cindea@math.univ-bpclermont.fr, arnaud.munch@math.univ-bpclermont.fr.
} 
The null controllability problem for (1) at time $T$ is the following: for each $\left(y_{0}, y_{1}\right) \in \boldsymbol{V}$, find $v \in L^{2}\left(q_{T}\right)$ such that the corresponding solution to (1) satisfies

$$
y(\cdot, T)=0, \quad y_{t}(\cdot, T)=0 \quad \text { in }(0,1) .
$$

When the subset $q_{T}$ takes the form $q_{T}:=\omega \times(0, T)$, where $\omega$ denotes a subset of $(0,1)$, the null-controllability of (1) at any large time $T>T^{\star}$ is well-known (for instance, see [1, 19]). The critical time $T^{\star}$ is related to the measure of $(0,1) \backslash \omega$. Moreover, as a consequence of the Hilbert Uniqueness Method of J.-L. Lions [19], the null controllability of (1) is equivalent to an observability inequality for the associated adjoint problem : there exists $C>0$ such that

$$
\left\|\varphi(\cdot, 0), \varphi_{t}(\cdot, 0)\right\|_{\boldsymbol{H}}^{2} \leq C\|\varphi\|_{L^{2}(\omega \times(0, T))}^{2}, \quad \forall\left(\varphi_{0}, \varphi_{1}\right) \in \boldsymbol{H}:=L^{2}(0,1) \times H^{-1}(0,1)
$$

where $\left(\varphi, \varphi_{0}, \varphi_{1}\right)$ solves

$$
L \varphi=0 \quad \text { in } \quad Q_{T}, \quad \varphi=0 \quad \text { on } \quad \Sigma_{T} ; \quad\left(\varphi(\cdot, 0), \varphi_{t}(\cdot, 0)\right)=\left(\varphi_{0}, \varphi_{1}\right) \quad \text { in } \quad(0,1) .
$$

We investigate in this work some questions related to the controllability of (1) for more general subsets $q_{T} \subset Q_{T}$ where the support of the control function $v$ depends on the time variable. A particular example is to consider the region between two curves, i.e.

$$
q_{T}=\left\{(x, t) \in Q_{T} ; a(t)<x<b(t), t \in(0, T)\right\} .
$$

A geometrical description is given by Figure 1.

To our knowledge, the control of PDEs with non-cylindrical support has been much less addressed in the literature. For the wave equation, we mention the contribution of Khapalov [18] providing observability results for a moving point sensor in the interior of the domain, allowing the author to avoid the usual difficulties related to strategic or non-strategic points. In particular, in the $1 \mathrm{D}$ setting, for any $T>0$, the existence of controls continuous almost everywhere in $(0, T)$, supported over curves continuous almost everywhere is obtained for data in $H^{2}(0,1) \cap H_{0}^{1}(0,1) \times H_{0}^{1}(0,1)$. More recently, let us mention two works concerning again the $1 \mathrm{D}$ wave equation both for initial data in $\boldsymbol{H}$ and any $T>2$ : the first one [6] analyzes the exact controllability from a moving interior point. By the way of the d'Alembert formula, an observability inequality is proved for a precise sets of curves $\{(\gamma(t), t)\}_{t \in(0, T)}$ leading to moving controls in $H^{-1}\left(\cup_{t \in(0, T)} \gamma(t) \times\{t\}\right)$. The second one [11] considers the controllability from the moving boundary of the form $1+k t$ with $k \in(0,1], t \in(0, T)$. In the case $k<1$, the controllability in $L^{2}(0, T)$ is proved by the way of the multiplier method: as is usual, a change of variable reduces the problem on fixed domains for wave equation with non constants coefficient. In the case $k=1$ for which the speed of the moving endpoint is equal to the characteristic speed, the d'Alembert formula allows to characterize the reachable set. Eventually, we mention that in [20], the authors prove that a mobile control support in time allows to recover the controllability of the damped wave equation $y_{t t}-y_{x x}-\varepsilon y_{t x x}=0$ defined on the $1 \mathrm{D}$ torus, uniformly with respect to the parameter $\varepsilon>0$ (due the appearance of an essential spectrum as $\varepsilon \rightarrow 0$, the uniform controllability property can not be obtained if the control simply acts on a cylindrical domain).

Let us denote by $\mathcal{C}(a, b, T)$ the class of domains of the form given by 6 for which the controllability holds, or equivalently the set of triplet $(a, b, T) \in C([0, T]] 0,,1[) \times C([0, T]] 0,,1[) \times \mathbf{R}^{+}$for which the controllability hold. Obviously, this set is not empty: it suffices that $T$ be large enough and that the domain $\left\{(x, t) \in Q_{T} ; a(t)<x<b(t), t \in(0, T)\right\}$ contains any rectangular domain $\left(a_{1}, b_{1}\right) \times(0, T)$ (i.e. that there exists $a_{1}>0, b_{1}>0$ such that $a(t) \leq a_{1}<b_{1} \leq b(t)$ for all time $t \in[0, T])$ and then to apply (4).

The first contribution of this work is the extension of the well-known observability inequality (4) to time-depending subsets $q_{T}$, as for instance those given by (6). Precisely, we prove that if 
$T>2$ and any characteristic line starting at any point $x \in(0,1)$ at time $t=0$ and following the optical geometric conditions when reflecting at the boundary $x=0,1$, meets the observation subset $q_{T}$ then the following estimate turns out to be true :

$$
\left.\| \varphi(\cdot, 0), \varphi_{t}(\cdot, 0)\right) \|_{\boldsymbol{H}}^{2} \leq C\left(\|\varphi\|_{L^{2}\left(q_{T}\right)}^{2}+\|L \varphi\|_{L^{2}\left(0, T ; H^{-1}(0,1)\right)}^{2}\right), \quad \forall \varphi \in \Phi
$$

where $\Phi=\left\{\varphi: \varphi \in L^{2}\left(q_{T}\right), \varphi=0\right.$ on $\Sigma_{T}$ such that $\left.L \varphi \in L^{2}\left(0, T ; H^{-1}(0,1)\right)\right\}$. This so-called generalized inequality observability implies by duality arguments the null controllability of (1) with controls in $L^{2}\left(q_{T}\right)$ for $q_{T}$ given by (6).

The second contribution of this work is the introduction of a numerical method for the approximation of the control of minimal $L^{2}\left(q_{T}\right)$-norm. Usually (for instance see [16, 17, 22]), such approximation is based on the minimization of the so-called conjugate functional :

$$
\min _{\left(\varphi_{0}, \varphi_{1}\right) \in \boldsymbol{H}} J^{\star}\left(\varphi_{0}, \varphi_{1}\right)=\frac{1}{2} \iint_{q_{T}}|\varphi|^{2} d x d t+<\varphi_{1}, y_{0}>_{H^{-1}(0,1), H_{0}^{1}(0,1)}-\int_{0}^{1} \varphi_{0} y_{1} d x
$$

where $\left(\varphi, \varphi_{0}, \varphi_{1}\right)$ solves (5). Here and in the sequel, we use the following duality pairing:

$$
<\varphi_{1}, y_{0}>_{H^{-1}(0,1), H_{0}^{1}(0,1)}=\int_{0}^{1} \partial_{x}\left((-\Delta)^{-1} \varphi_{1}\right)(x) \partial_{x} y_{0}(x) d x
$$

where $-\Delta$ is the Dirichlet Laplacian in $(0,1)$. The well-posedness of this extremal problem precisely the coercivity of $J^{\star}$ over $\boldsymbol{H}$ - is given by the estimate $(7)$. The control of minimal $L^{2}\left(q_{T}\right)$ norm is then given by $v=-\varphi 1_{q_{T}}$ where $\varphi$ solves (5) associated to $\left(\varphi_{0}, \varphi_{1}\right)$, unique minimizer of $J^{\star}$. Since the domain $q_{T}$ evolves in time, the resolution of the wave equation by any usual time marching method leads to some technicalities (at the level of the numerical implementation). We therefore use, in the spirit of the works [9, 10], an equivalent formulation where the time and the space variables are embedded in a time-space variational formulation, very appropriate to our non-cylindrical situation. Specifically, we consider the following extremal problem over the variable $\varphi$ :

$$
\min _{\varphi \in W} \hat{J}^{\star}(\varphi)=\frac{1}{2} \iint_{q_{T}}|\varphi|^{2} d x d t+<\varphi_{1}, y_{0}>_{H^{-1}(0,1), H_{0}^{1}(0,1)}-\int_{0}^{1} \varphi_{0} y_{1} d x,
$$

with $W=\left\{\varphi: \varphi \in L^{2}\left(q_{T}\right), \varphi=0\right.$ on $\Sigma_{T}$ such that $\left.L \varphi=0 \in L^{2}\left(0, T ; H^{-1}(0,1)\right)\right\}$. The unknown is now the whole solution $\varphi$, constrained by the equality $L \varphi=0$ in $L^{2}\left(0, T ; H^{-1}(0,1)\right)$. This constraint is taken into account with a Lagrangian multiplier $\lambda \in L^{2}\left(0, T ; H_{0}^{1}(0,1)\right)$ through a mixed formulation in $(\varphi, \lambda)$ solved using a conformal finite element approximation in time and space.

This paper is organized as follows. In Section 2, we prove the generalized observability inequality (7) over the Hilbert space $\Phi$ (see Proposition 2.1) leading to the uniform controllability of (1) in $\boldsymbol{V}$. In Section 3, we adapt 10 and introduce the mixed formulation $(24)$ in the variable $(\varphi, \lambda) \in$ $\Phi \times L^{2}\left(0, T ; H_{0}^{1}(0,1)\right)$ equivalent to the extremal problem $(9)$. The variable $\lambda$ can be interpreted as a Lagrange multiplier for the equality constraint $L \varphi=0$ in $L^{2}\left(0, T ; H^{-1}(0,1)\right)$. We employ the estimate (7) to prove the well-posedness of this mixed formulation. In particular, we prove an inf-sup condition for the pair $(\varphi, \lambda)$. Moreover, it turns out that the multiplier $\lambda$, unique solution of the mixed formulation, coincides with the controlled state $y$, solution of (1) (in the weak-sense) (see section 3.1. This property allows to define Section 3.2 an another equivalent extremal problem (the so-called primal problem, dual of the problem (9)) in the controlled solution $y$ only (see Proposition 3.1), without the introduction of any penalty parameter. The corresponding elliptic problem in $L^{2}\left(0, T ; H_{0}^{1}(0,1)\right)$ is solved by the way of a conjugate gradient algorithm. Section 4 is devoted to the numerical approximation of the mixed formulation as well as some numerical experiments. We emphasize the robustness of the approach leading notably to the strong convergence of discrete 
sequence $\left\{v_{h}\right\}$ toward the controls for various geometries of $q_{T}$. Eventually, Section 5 concludes with some perspectives: in particular, we highlight the natural extension of this work which consists in optimizing the control of (1) with respect to the support $q_{T}$.
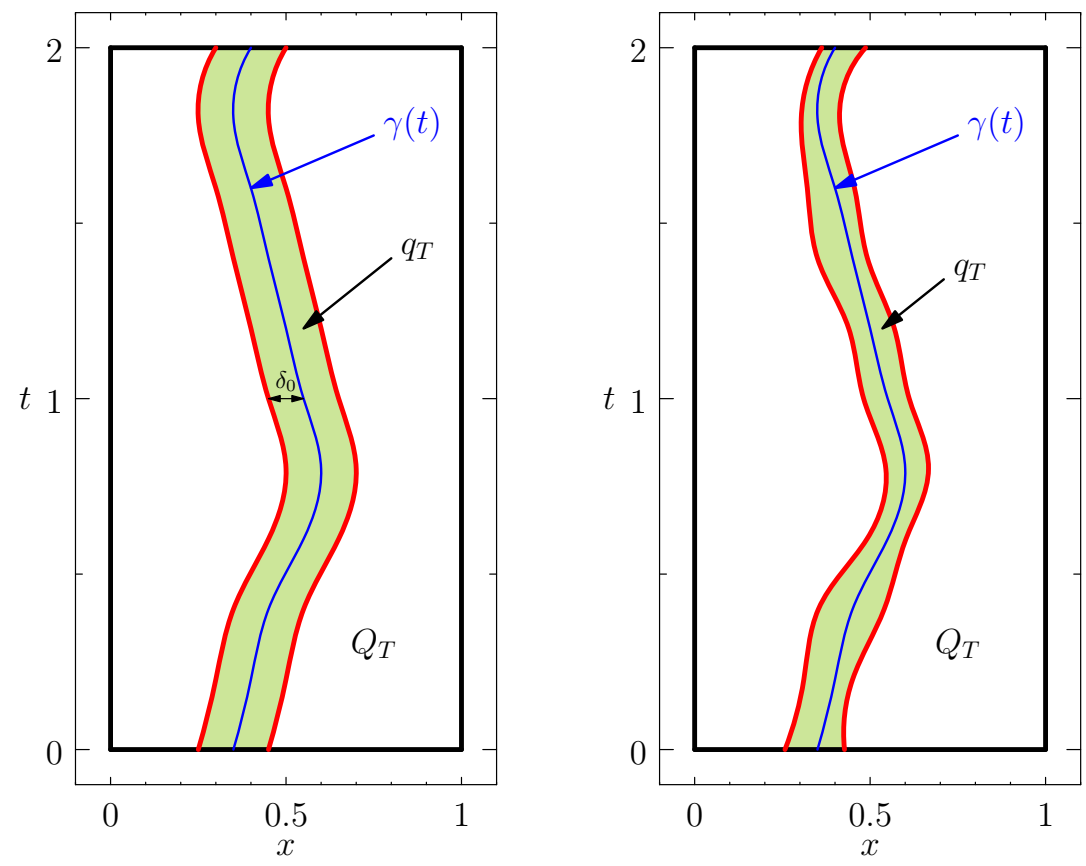

Figure 1: Time dependent domains $q_{T}$ included in $Q_{T}$.

\section{$2 \quad$ A generalized observability inequality}

Let $q_{T} \subset(0,1) \times(0, T)$ be an open set. We define the vectorial space

$$
\Phi=\left\{\varphi: \varphi \in L^{2}\left(q_{T}\right), \varphi=0 \text { on } \Sigma_{T} \text { such that } L \varphi \in L^{2}\left(0, T ; H^{-1}(0,1)\right)\right\} .
$$

Endowed with the following inner product

$$
(\varphi, \bar{\varphi})_{\Phi}=\iint_{q_{T}} \varphi(x, t) \bar{\varphi}(x, t) d x d t+\eta \int_{0}^{T}<L \varphi, L \bar{\varphi}>_{H^{-1}(0,1), H^{-1}(0,1)} d t
$$

for any fixed $\eta>0$, the space $\Phi$ is an Hilbert space.

In this section, we prove the following result.

Proposition 2.1 Assume that $T>2$ and $q_{T} \subset(0,1) \times(0, T)$ is a finite union of connected open sets and satisfies the following hypotheses: Any characteristic line starting at the point $x \in(0,1)$ at time $t=0$ and following the optical geometric laws when reflecting at the boundaries $x=0,1$ must meet $q_{T}$.

Then, there exists $C>0$ such that the following estimate holds :

$$
\left.\| \varphi(\cdot, 0), \varphi_{t}(\cdot, 0)\right) \|_{\boldsymbol{H}}^{2} \leq C\left(\|\varphi\|_{L^{2}\left(q_{T}\right)}^{2}+\|L \varphi\|_{L^{2}\left(0, T ; H^{-1}(0,1)\right)}^{2}\right), \quad \forall \varphi \in \Phi .
$$

PROOF: We proceed in several steps: 
Step 1: Let $\varphi$ be a smooth solutions of the wave equation (5). Note that $\varphi$ can be extended in a unique way to a function, still denoted by $\varphi$, in $(x, t) \in(0,1) \times \mathbf{R}$ satisfying $L \varphi=0$ and the boundary conditions $\varphi(0, t)=\varphi(1, t)=0$ for all $t \in \mathbf{R}$. In this first step we prove that for such extension the following holds: For each $t \in \mathbf{R}, x \in(0,1)$ and $\delta>0$

$$
\begin{aligned}
\int_{t-\delta}^{t+\delta} \varphi_{x}(0, s) d s & \leq \frac{1}{\delta} \iint_{\mathcal{U}_{(x, t+x)}^{\delta}}\left(\left|\varphi_{x}(y, s)\right|^{2}+\left|\varphi_{t}(y, s)\right|^{2}\right) d y d s \\
\int_{t-\delta}^{t+\delta} \varphi_{x}(0, s) d s & \leq \frac{1}{\delta} \iint_{\mathcal{U}_{(x, t-x)}^{\delta}}\left(\left|\varphi_{x}(y, s)\right|^{2}+\left|\varphi_{t}(y, s)\right|^{2}\right) d y d s,
\end{aligned}
$$

where $\mathcal{U}_{(x, t)}^{\delta}$ is a neighborhood of $(x, t)$ of the form $\mathcal{U}_{(x, t)}^{\delta}=\{(y, s)$ such that $|x-y|+|t-s|<\delta\}$.

Let us start with (12). Note that wave equation is symmetric with respect to the time and space variables. Therefore, the D'Alembert formulae can be used changing the time and space role, i.e.

$$
\frac{1}{2} \int_{t-x}^{t+x} u_{x}(0, s) d s=u(x, t), \quad(x, t) \in(0,1) \times \mathbf{R},
$$

where we have taken into account the boundary condition $u(0, t)=0$. Consider now $x=x_{0}-t$ in (14) and differentiate with respect to time. Then,

$$
-\varphi_{x}\left(0,2 t-x_{0}\right)=-\varphi_{x}\left(x_{0}-t, t\right)+\varphi_{t}\left(x_{0}-t, t\right)
$$

that written in the original variables $(x, t)$ gives,

$$
\varphi_{x}(0, t-x)=\varphi_{x}(x, t)-\varphi_{t}(x, t)
$$

or equivalently

$$
\varphi_{x}(0, t)=\varphi_{x}(x, t+x)-\varphi_{t}(x, t+x), \quad t \in \mathbf{R}, \quad x \in(0,1) .
$$

Integrating the square of 15 in $(y, s) \in \mathcal{U}_{(x, t+x)}^{\delta}$ with the parametrization

$$
\left\{\begin{array}{l}
y=x+\frac{u-v}{\sqrt{2}} \\
s=t+x+\frac{u+v}{\sqrt{2}}
\end{array}, \quad|u|,|v|<\delta / \sqrt{2}\right.
$$

we obtain

$$
\int_{-\delta / \sqrt{2}}^{\delta / \sqrt{2}} \int_{-\delta / \sqrt{2}}^{\delta / \sqrt{2}}\left|u_{x}(0, t+2 v / \sqrt{2})\right|^{2} d u d v=\iint_{\mathcal{U}_{x, t+x}^{\delta}}\left|u_{x}(y, s)-u_{t}(y, s)\right|^{2} d y d s .
$$

Therefore, with the change $s=t+2 v / \sqrt{2}$ in the first integral, we easily obtain 12. Formula (13) is obtained in a similar way but this time with the change $x=x_{0}+t$ in (14).

Step 2. Here we show that there exists a constant $C>0$ such that,

$$
\left.\| \varphi(\cdot, 0), \varphi_{t}(\cdot, 0)\right) \|_{\boldsymbol{V}}^{2} \leq C\left(\left\|\varphi_{t}\right\|_{L^{2}\left(q_{T}\right)}^{2}+\left\|\varphi_{x}\right\|_{L^{2}\left(q_{T}\right)}^{2}\right),
$$

for any $\varphi \in W$ and initial data in $\boldsymbol{V}$.

We may assume that $\varphi$ is smooth since the general case can be obtained by a usual density argument. We also assume that $\varphi$ is extended to $(x, t) \in(0,1) \times \mathbf{R}$ by assuming that it satisfies the wave equation and boundary conditions in this region. This extension is unique and 2-periodic in time. The region $q_{T}$ is also extended to $\tilde{q}_{T}$ to take advantage of the time periodicity of the solution $\varphi$. We define,

$$
\tilde{q}_{T}=\bigcup_{k \in \mathbb{Z}}\left\{(x, t) \quad \text { such that }(x, t+2 k) \in q_{T}\right\} .
$$


The key point now is to observe that the hypotheses on $q_{T}$, namely the fact that any characteristic line starting at point $(x, 0)$ and following the optical geometric laws when reflecting at the boundary must meet $q_{T}$, is equivalent to the following: For any point $(0, t)$ with $t \in[0, T]$ there exists one characteristic line (either $(x, t+x)$ with $x \in(0,1)$ or $(x, t-x)$ ) that meets $\tilde{q}_{T}$.

Thus, given $t \in[0, T]$ we can apply either $\left[12\right.$ with $(x, t+x) \in \tilde{q}_{T}$ or $\left[13\right.$ with $(x, t-x) \in \tilde{q}_{T}$. Moreover, as $\tilde{q}_{T}$ is an open set, we may assume $\delta$ sufficiently small so that the neighborhoods $\mathcal{U}_{(x, t+x)}^{\delta} \subset \tilde{q}_{T}$ or $\mathcal{U}_{(x, t-x)}^{\delta} \subset \tilde{q}_{T}$. In particular we see that for any $t \in[0, T]$ there exists $\delta_{t}>0$ and $C_{t}>0$ such that

$$
\int_{t-\delta_{t}}^{t+\delta_{t}}\left|\varphi_{x}(0, s)\right|^{2} d s \leq C_{t} \iint_{\tilde{q}_{T}}\left(\left|\varphi_{t}\right|^{2}+\left|\varphi_{x}\right|^{2}\right) d x d t
$$

By compacity, there exists a finite number of times $t_{1}, \ldots, t_{n}$ such that $\cup_{i=1, \ldots, n}\left(t_{i}-\delta_{t_{i}}, t_{i}+\delta_{t_{i}}\right)$ covers the whole interval $[0, T]$ and therefore, by adding the corresponding inequalities, there exists $C>0$ such that

$$
\int_{0}^{T}\left|\varphi_{x}(0, s)\right|^{2} d s \leq C \iint_{\tilde{q}_{T}}\left(\left|\varphi_{t}\right|^{2}+\left|\varphi_{x}\right|^{2}\right) d x d t .
$$

The fact that we can replace $\tilde{q}_{T}$ by $q_{T}$ is easily checked by the 2-periodicity of $\varphi$ in time. Finally, it remains to see that we can replace the left hand side in $(17)$ by $\left.\| \varphi(\cdot, 0), \varphi_{t}(\cdot, 0)\right) \|_{\boldsymbol{V}}^{2}$, but this is a consequence of the well-known boundary observability inequality (see for instance [19])

$$
\left.\| \varphi(\cdot, 0), \varphi_{t}(\cdot, 0)\right) \|_{\boldsymbol{V}}^{2} \leq C \int_{0}^{T}\left|\varphi_{x}(0, s)\right|^{2} d s
$$

which holds for $T>2$.

Step 3. Here we show that we can substitute $\varphi_{x}$ by $\varphi$ in the right hand side of (16), i.e.

$$
\left.\| \varphi(\cdot, 0), \varphi_{t}(\cdot, 0)\right) \|_{\boldsymbol{V}}^{2} \leq C\left(\left\|\varphi_{t}\right\|_{L^{2}\left(q_{T}\right)}^{2}+\|\varphi\|_{L^{2}\left(q_{T}\right)}^{2}\right),
$$

for any $\varphi \in W$ and initial data in $\boldsymbol{V}$. In fact, this requires to extend slightly the observation zone $q_{T}$. Instead, we observe that if $q_{T}$ satisfies the hypotheses in Proposition 2.1 then there exists a smaller open subset $\tilde{q}_{T} \subset q_{T}$ that still satisfies the same hypotheses and such that the closure of $\tilde{q}_{T}$ is included in $q_{T}$. Thus, 16 must hold as well for $\tilde{q}_{T}$. Let us introduce now a function $\eta \geq 0$ which satisfies the following hypotheses:

$$
\begin{gathered}
\eta \in C^{1}((0,1) \times(0, T)), \quad \operatorname{supp}(\eta) \subset q_{T}, \quad\left\|\eta_{t}\right\|_{L^{\infty}}+\left\|\eta_{x}^{2} / \eta\right\|_{L^{\infty}} \leq C_{1} \text { in } q_{T} \\
\eta>\eta_{0}>0 \text { in } \tilde{q}_{T}, \text { with } \eta_{0}>0 \text { constant. }
\end{gathered}
$$

As $q_{T}$ is a finite union of connected open sets, the function $\eta$ can be easily obtained by convolution of the characteristic function of $\tilde{q}_{T}$ with a positive mollifier.

Multiplying the equation of $\varphi$ by $\eta \varphi$ and integrating by parts we easily obtain

$$
\begin{aligned}
\iint_{q_{T}} \eta\left|\varphi_{x}\right|^{2} d x d t & =\iint_{q_{T}} \eta\left|\varphi_{t}\right|^{2} d x d t+\iint_{q_{T}}\left(\eta_{t} \varphi \varphi_{t}-\eta_{x} \varphi \varphi_{x}\right) d x d t \\
\leq & \iint_{q_{T}} \eta\left|\varphi_{t}\right|^{2} d x d t+\frac{\left\|\eta_{t}\right\|_{L^{\infty}\left(q_{T}\right)}}{2} \iint_{q_{T}}\left(|\varphi|^{2}+\left|\varphi_{t}\right|\right) d x d t \\
& +\frac{1}{2} \iint_{q_{T}}\left(\frac{\eta_{x}^{2}}{\eta} \varphi^{2}+\eta \varphi_{x}^{2}\right) d x d t .
\end{aligned}
$$

Therefore,

$$
\iint_{q_{T}} \eta\left|\varphi_{x}\right|^{2} d x d t \leq C \iint_{q_{T}}\left(\left|\varphi_{t}\right|^{2}+|\varphi|^{2}\right) d x d t
$$


for some constant $C>0$, and we obtain

$$
\left\|\varphi_{x}\right\|_{L^{2}\left(\tilde{q}_{T}\right)}^{2} \leq C_{2}^{-1} \iint_{q_{T}} \eta\left|\varphi_{x}\right|^{2} d x d t \leq C_{2}^{-1} C \iint_{q_{T}}\left(\left|\varphi_{t}\right|^{2}+|\varphi|^{2}\right) d x d t .
$$

This combined with 160 for $\tilde{q}_{T}$ provides 18 .

Step 4. Here we prove that we can remove the second term in the right hand side of (18), i.e.

$$
\left.\| \varphi(\cdot, 0), \varphi_{t}(\cdot, 0)\right)\left\|_{\boldsymbol{V}}^{2} \leq C\right\| \varphi_{t} \|_{L^{2}\left(q_{T}\right)}^{2}
$$

for any $\varphi \in W$ and initial data in $\boldsymbol{V}$. Note that, for each time $t \in[0, T]$ and each $\omega \subset \Omega$ we have the following regularity estimate

$$
\left.\int_{a(t)}^{b(t)}|\varphi(x, t)|^{2} d x \leq \| \varphi(\cdot, 0), \varphi_{t}(\cdot, 0)\right) \|_{\boldsymbol{H}}^{2}, \quad \text { for all } t \in[0, T]
$$

Therefore, integrating in time, we easily obtain

$$
\left.\|\varphi\|_{L^{2}\left(q_{T}\right)}^{2} \leq T \| \varphi(\cdot, 0), \varphi_{t}(\cdot, 0)\right) \|_{\boldsymbol{H}}^{2} .
$$

We now substitute this inequality in 16

$$
\left.\left.\| \varphi(\cdot, 0), \varphi_{t}(\cdot, 0)\right)\left\|_{\boldsymbol{V}}^{2} \leq C\left(\left\|\varphi_{t}\right\|_{L^{2}\left(q_{T}\right)}^{2}+\| \varphi(\cdot, 0), \varphi_{t}(\cdot, 0)\right)\right\|_{\boldsymbol{H}}^{2}\right) .
$$

Inequality $(19)$ is finally obtained by contradiction. Assume that it is not true. Then, there exists a sequence $\left.\left(\varphi^{k}(\cdot, 0), \varphi_{t}^{k}(\cdot, 0)\right)\right)_{k>0} \in \boldsymbol{V}$ such that

$$
\begin{aligned}
& \left.\| \varphi^{k}(\cdot, 0), \varphi_{t}^{k}(\cdot, 0)\right) \|_{\boldsymbol{V}}^{2}=1, \quad \forall k>0 \\
& \left\|\varphi_{t}^{k}\right\|_{L^{2}\left(q_{T}\right)}^{2} \rightarrow 0, \text { as } k \rightarrow \infty .
\end{aligned}
$$

Therefore, there exists a subsequence, still denoted by the index $k$, such that $\left(\varphi^{k}(\cdot, 0), \varphi_{t}^{k}(\cdot, 0)\right) \rightarrow$ $\left(\varphi^{\star}(\cdot, 0), \varphi_{t}^{\star}(\cdot, 0)\right)$ weakly in $\boldsymbol{V}$ and strongly in $\boldsymbol{H}$ (by the compactness of the inclusion $\boldsymbol{V} \subset \boldsymbol{H}$ ). Passing to the limit in the equation we easily see that the solution associated to $\left(\varphi^{\star}(\cdot, 0), \varphi_{t}^{\star}(\cdot, 0)\right)$, $\varphi^{\star}$ must vanish at $q_{T}$ and therefore, by $(18), \varphi^{\star}=0$. In particular this is in contradiction with 200 since it implies that the right hand side in 20) vanishes as $k \rightarrow \infty$ while the left hand side is bounded by below.

Step 5. We now write 19 with respect to the weaker norm. In particular, we obtain

$$
\left.\| \varphi(\cdot, 0), \varphi_{t}(\cdot, 0)\right)\left\|_{\boldsymbol{H}}^{2} \leq C\right\| \varphi \|_{L^{2}\left(q_{T}\right)}^{2},
$$

for any $\varphi \in \Phi$ with $L \varphi=0$.

Let $\eta \in \Phi$ be the solution of $L \eta=0$ and initial data $\left(\Delta^{-1} \varphi_{t}(\cdot, 0), \varphi(\cdot, 0)\right) \in \boldsymbol{V}$ where $\Delta$ designates the Dirichlet Laplacian in $(0,1)$. Let us write $\eta(x, t)=\eta(x, 0)+\int_{0}^{t} \varphi(x, s) d s$, for all $(x, t) \in Q_{T}$. Then, inequality 19 on $\eta$ and the fact that $\Delta$ is an isomorphism from $H_{0}^{1}(0,1)$ to $H^{-1}(0,1)$, provide

$$
\begin{aligned}
\left\|\left(\varphi(\cdot, 0), \varphi_{t}(\cdot, 0),\right)\right\|_{\boldsymbol{H}}^{2} & =\left\|\left(\Delta^{-1} \varphi_{t}(\cdot, 0), \varphi(\cdot, 0)\right)\right\|_{\boldsymbol{V}}^{2} \\
& \leq C\left\|\eta_{t}\right\|_{L^{2}\left(q_{T}\right)}^{2}=C\|\varphi\|_{L^{2}\left(q_{T}\right)}^{2} .
\end{aligned}
$$

Step 6. Here we finally obtain (11). Given $\varphi \in \Phi$ we can decompose it as $\varphi=\varphi_{1}+\varphi_{2}$ where $\varphi_{1}, \varphi_{2} \in \Phi$ solve

$$
\left\{\begin{array} { l } 
{ L \varphi _ { 1 } = L \varphi , } \\
{ \varphi _ { 1 } ( \cdot , 0 ) = ( \varphi _ { 1 } ) _ { t } ( \cdot , 0 ) = 0 }
\end{array} \quad \left\{\begin{array}{l}
L \varphi_{2}=0, \\
\varphi_{2}(\cdot, 0)=\varphi(\cdot, 0), \quad\left(\varphi_{2}\right)_{t}(\cdot, 0)=\varphi_{t}(\cdot, 0) .
\end{array}\right.\right.
$$


From Duhamel's principle, we can write

$$
\varphi_{1}(\cdot, t)=\int_{0}^{t} \psi(\cdot, t-s, s) d s
$$

where $\psi(x, t, s)$ solves, for each value of the parameter $s \in(0, t)$,

$$
\left\{\begin{array}{l}
L \psi(\cdot, \cdot, s)=0, \\
\psi(\cdot, 0, s)=0, \quad \psi_{t}(\cdot, 0, s)=L \varphi(\cdot, s)
\end{array}\right.
$$

Therefore,

$$
\begin{aligned}
& \left.\left\|\varphi_{1}\right\|_{L^{2}\left(q_{T}\right)}^{2} \leq \int_{0}^{T}\|\psi(\cdot, \cdot, s)\|_{L^{2}\left(q_{T}\right)}^{2} d s \leq C \int_{0}^{T} \| \psi(\cdot, 0, s), \psi_{t}(\cdot, 0, s)\right) \|_{\boldsymbol{H}^{2}}^{2} d s \\
& \leq C\|L \varphi\|_{L^{2}\left(0, T ; H^{-1}(0,1)\right)}^{2}
\end{aligned}
$$

Combining 222 and estimate 21] for $\varphi_{2}$ we obtain

$$
\begin{aligned}
& \left.\left.\| \varphi(\cdot, 0), \varphi_{t}(\cdot, 0)\right)\left\|_{\boldsymbol{H}}^{2}=\right\| \varphi_{2}(\cdot, 0),\left(\varphi_{2}\right)_{t}(\cdot, 0)\right)\left\|_{\boldsymbol{H}}^{2} \leq C\right\| \varphi_{2} \|_{L^{2}\left(q_{T}\right)}^{2} \\
& \leq C\left(\|\varphi\|_{L^{2}\left(q_{T}\right)}^{2}+\left\|\varphi_{1}\right\|_{L^{2}\left(q_{T}\right)}^{2}\right) \leq C\left(\|\varphi\|_{L^{2}\left(q_{T}\right)}^{2}+\|L \varphi\|_{L^{2}\left(0, T ; H^{-1}\right)}^{2}\right) .
\end{aligned}
$$

Remark 1 1. The hypotheses on $q_{T}$ stated in Proposition 2.1 are optimal in the following sense: If there exists a subinterval $\omega_{0} \subset(0,1)$ for which all characteristics starting in $\omega_{0}$ and following the geometrical optics conditions when getting to the boundary $x=0,1$, do not meet $q_{T}$, then the inequality fails to hold. This is easily seen by considering particular solutions of the wave equation which initial data supported in $\omega_{0}$.

2. The proof of inequality (11) above does not provide an estimate on the dependence of the constant with respect to $q_{T}$.

3. In the cylindrical situation, i.e. $q_{T}=(a, b) \times(0, T)$, a generalized Carleman inequality, valid for the wave equation with variable coefficients, have been obtained in [9] (see also [26]). To our knowledge, the extension of Proposition 2.1 to the wave equation with variable coefficients is still open and a priori can not be obtained by the method used in this section.

\section{Control of minimal $L^{2}\left(q_{T}\right)$-norm: a mixed reformulation}

We now adapt in this section the work [10] and present a mixed formulation based on the optimality conditions associated to the extremal problem (8) (section 3.1). From a numerical point of view, this mixed formulation is more appropriate to the non-cylindrical situation considered in this work.

\subsection{Mixed reformulation of the controllability problem}

As described at length in [10, the starting point of the reformulation is the dual problem (8). Thus, in order to avoid the minimization of the functional $J^{\star}$ with respect to the initial data $\left(\varphi_{0}, \varphi_{1}\right)$, we now present a direct way to approximate the control of minimal square integrable norm, in the spirit of the primal approach developed in 9 . Since the variable $\varphi$, solution of (5), is completely and uniquely determined by the data $\left(\varphi_{0}, \varphi_{1}\right)$, the main idea of the reformulation is to keep $\varphi$ as main variable and consider the following extremal problem:

$$
\min _{\varphi \in W} \hat{J}^{\star}(\varphi)=\frac{1}{2} \iint_{q_{T}}|\varphi|^{2} d x d t+<\varphi_{t}(\cdot, 0), y_{0}>_{H^{-1}(0,1), H_{0}^{1}(0,1)}-\int_{0}^{1} \varphi(\cdot, 0) y_{1} d x
$$


where

$$
W=\left\{\varphi: \varphi \in L^{2}\left(q_{T}\right), \varphi=0 \text { on } \Sigma_{T} \text { such that } L \varphi=0\right\},
$$

endowed with the same inner product than $\Phi$, given in 100 .

Remark that from (11) the property $\varphi \in W$ implies that $\left(\varphi(\cdot, 0), \varphi_{t}(\cdot, 0)\right) \in \boldsymbol{H}$, so that the functional $\hat{J}^{\star}$ is well-defined over $W$. Therefore, the minimization of $\hat{J}^{\star}$ is evidently equivalent to the minimization of $J^{\star}$ over $\boldsymbol{H}$.

The main variable is now $\varphi$ submitted to the constraint equality $L \varphi=0$ as an $L^{2}\left(0, T ; H^{-1}(0,1)\right)$ function. This constraint is addressed introducing a Lagrangian multiplier $\lambda \in L^{2}\left(0, T ; H_{0}^{1}(\Omega)\right)$ as follows:

We consider the following problem : find $(\varphi, \lambda) \in \Phi \times L^{2}\left(0, T ; H_{0}^{1}(0,1)\right)$ solution of

$$
\left\{\begin{aligned}
a(\varphi, \bar{\varphi})+b(\bar{\varphi}, \lambda) & =l(\bar{\varphi}), & & \forall \bar{\varphi} \in \Phi \\
b(\varphi, \bar{\lambda}) & =0, & & \forall \bar{\lambda} \in L^{2}\left(0, T ; H_{0}^{1}(0,1)\right),
\end{aligned}\right.
$$

where

$$
\begin{array}{r}
a: \Phi \times \Phi \rightarrow \mathbb{R}, \quad a(\varphi, \bar{\varphi})=\iint_{q_{T}} \varphi \bar{\varphi} d x d t \\
\begin{array}{r}
b: \Phi \times L^{2}\left(0, T ; H_{0}^{1}(0,1)\right) \rightarrow \mathbb{R}, \quad b(\varphi, \lambda) \\
=\int_{0}^{T}<L \varphi, \lambda>_{H^{-1}(0,1), H_{0}^{1}(0,1)} d t \\
\quad=\iint_{Q_{T}} \partial_{x}\left(-\Delta^{-1}(L \varphi)\right) \cdot \partial_{x} \lambda d x d t
\end{array} \\
l: \Phi \rightarrow \mathbb{R}, \quad l(\varphi)=-<\varphi_{t}(\cdot, 0), y_{0}>_{H^{-1}(0,1), H_{0}^{1}(0,1)}+\int_{0}^{1} \varphi(\cdot, 0) y_{1} d x .
\end{array}
$$

We have the following result :

TheORem 3.1 Under the hypothesis of Proposition 2.1, we have:

(i) The mixed formulation (24) is well-posed.

(ii) The unique solution $(\varphi, \lambda) \in \Phi \times L^{2}\left(0, T ; H_{0}^{1}(0,1)\right)$ is the unique saddle-point of the Lagrangian $\mathcal{L}: \Phi \times L^{2}\left(0, T ; H_{0}^{1}(0,1)\right) \rightarrow \mathbb{R}$ defined by

$$
\mathcal{L}(\varphi, \lambda)=\frac{1}{2} a(\varphi, \varphi)+b(\varphi, \lambda)-l(\varphi)
$$

(iii) The optimal function $\varphi$ is the minimizer of $\hat{J}^{\star}$ over $\Phi$ while the optimal function $\lambda \in$ $L^{2}\left(0, T ; H_{0}^{1}(0,1)\right)$ is the state of the controlled wave equation (1) in the weak sense (associated to the control $\left.-\varphi 1_{q_{T}}\right)$.

Proof - We easily check that the bilinear form $a$ is continuous over $\Phi \times \Phi$, symmetric and positive and that the bilinear form $b$ is continuous over $\Phi \times L^{2}\left(0, T ; H_{0}^{1}(0,1)\right)$. Furthermore, the continuity of the linear form $l$ over $\Phi$ is a direct consequence of the generalized observability inequality (11):

$$
|l(\varphi)| \leq\left\|\left(y_{0}, y_{1}\right)\right\|_{\boldsymbol{V}} \sqrt{C \max \left(1, \eta^{-1}\right)}\|\varphi\|_{\Phi}, \quad \forall \varphi \in \Phi .
$$

Therefore, the well-posedness of the mixed formulation is a consequence of the following two properties (see [5]):

- $a$ is coercive on $\mathcal{N}(b)$, where $\mathcal{N}(b)$ denotes the kernel of $b$ :

$$
\mathcal{N}(b)=\left\{\varphi \in \Phi \text { such that } b(\varphi, \lambda)=0 \text { for every } \lambda \in L^{2}\left(0, T ; H_{0}^{1}(0,1)\right)\right\} ;
$$


- $b$ satisfies the usual "inf-sup" condition over $\Phi \times L^{2}\left(0, T ; H_{0}^{1}(0,1)\right)$ : there exists $\delta>0$ such that

$$
\inf _{\lambda \in L^{2}\left(0, T ; H_{0}^{1}(0,1)\right)} \sup _{\varphi \in \Phi} \frac{b(\varphi, \lambda)}{\|\varphi\|_{\Phi}\|\lambda\|_{L^{2}\left(0, T ; H_{0}^{1}(0,1)\right)}} \geq \delta .
$$

From the definition of $a$, the first point is clear : for all $\varphi \in \mathcal{N}(b)=W, a(\varphi, \varphi)=\|\varphi\|_{W}^{2}$. Let us check the inf-sup condition 26 . For any fixed $\lambda_{0} \in L^{2}\left(0, T ; H_{0}^{1}(0,1)\right)$, we define the (unique) element $\varphi_{0}$ such that $L \varphi_{0}=-\Delta \lambda_{0}$ in $Q_{T}$ and such that $\varphi_{0}(\cdot, 0)=0$ in $L^{2}(0,1)$ and $\varphi_{0, t}(\cdot, 0)=0$ in $H^{-1}(0,1) . \varphi_{0}$ is therefore solution of the wave equation with source term $-\Delta \lambda_{0} \in$ $L^{2}\left(0, T ; H^{-1}(0,1)\right)$, null Dirichlet boundary condition and zero initial state. We then use the following estimate (see for instance Chapter 1 in [19]): there exists a constant $C_{\Omega, T}>0$ such that

$$
\left\|\varphi_{0}\right\|_{L^{2}\left(Q_{T}\right)} \leq C_{\Omega, T}\left\|-\Delta \lambda_{0}\right\|_{L^{2}\left(0, T ; H^{-1}(0,1)\right)} \leq C_{\Omega, T}\left\|\lambda_{0}\right\|_{L^{2}\left(0, T ; H_{0}^{1}(0,1)\right)} .
$$

Consequently, $\varphi_{0} \in \Phi$. In particular, we have $b\left(\varphi_{0}, \lambda_{0}\right)=\left\|\lambda_{0}\right\|_{L^{2}\left(0, T ; H_{0}^{1}(0,1)\right)}^{2}$ and

$$
\begin{aligned}
\sup _{\varphi \in \Phi} \frac{b\left(\varphi, \lambda_{0}\right)}{\|\varphi\|_{\Phi}\left\|\lambda_{0}\right\|_{L^{2}\left(Q_{T}\right)}} \geq & \geq \frac{b\left(\varphi_{0}, \lambda_{0}\right)}{\left\|\varphi_{0}\right\|_{\Phi}\left\|\lambda_{0}\right\|_{L^{2}\left(Q_{T}\right)}} \\
& =\frac{\left\|\lambda_{0}\right\|_{L^{2}\left(0, T ; H_{0}^{1}(0,1)\right)}^{2}}{\left(\left\|\varphi_{0}\right\|_{L^{2}\left(q_{T}\right)}^{2}+\eta\left\|\lambda_{0}\right\|_{L^{2}\left(0, T ; H_{0}^{1}(0,1)\right)}^{2}\right)^{\frac{1}{2}}\left\|\lambda_{0}\right\|_{L^{2}\left(0, T ; H_{0}^{1}(0,1)\right)}} .
\end{aligned}
$$

Combining the above two inequalities, we obtain

$$
\sup _{\varphi_{0} \in \Phi} \frac{b\left(\varphi_{0}, \lambda_{0}\right)}{\left\|\varphi_{0}\right\|_{\Phi}\left\|\lambda_{0}\right\|_{L^{2}\left(0, T ; H_{0}^{1}(0,1)\right)}} \geq \frac{1}{\sqrt{C_{\Omega, T}^{2}+\eta}}
$$

and, hence, 26 holds with $\delta=\left(C_{\Omega, T}^{2}+\eta\right)^{-\frac{1}{2}}$.

The point (ii) is due to the symmetry and to the positivity of the bilinear form $a$. (iii). The equality $b(\varphi, \lambda)=0$ for all $\lambda \in L^{2}\left(0, T ; H_{0}^{1}(0,1)\right)$ implies that $L \varphi=0$ as an $L^{2}\left(0, T ; H^{-1}(0,1)\right)$ function, so that if $(\varphi, \lambda) \in \Phi \times L^{2}\left(0, T ; H_{0}^{1}(0,1)\right)$ solves the mixed formulation, then $\varphi \in W$ and $\mathcal{L}(\varphi, \lambda)=\hat{J}^{\star}(\varphi)$. Finally, the first equation of the mixed formulation reads as follows :

$$
\iint_{q_{T}} \varphi \bar{\varphi} d x d t+\int_{0}^{T}<L \bar{\varphi}, \lambda>_{H^{-1}, H_{0}^{1}} d t=l(\bar{\varphi}), \quad \forall \bar{\varphi} \in \Phi,
$$

or equivalently, since the control of minimal $L^{2}\left(q_{T}\right)$ norm is given by $v=-\varphi 1_{q_{T}}$,

$$
\iint_{Q_{T}}-v 1_{q_{T}} \bar{\varphi} d x d t+\int_{0}^{T}<L \bar{\varphi}, \lambda>_{H^{-1}, H_{0}^{1}} d t=l(\bar{\varphi}), \quad \forall \bar{\varphi} \in \Phi .
$$

But this means that $\lambda \in L^{2}\left(0, T, H_{0}^{1}(0,1)\right)$ is solution of the wave equation in the transposition sense. Since $\left(y_{0}, y_{1}\right) \in \boldsymbol{V}$ and $v \in L^{2}\left(q_{T}\right), \lambda$ must coincide with the unique weak solution to (1).

Therefore, Theorem 3.1 reduces the search of the control of square minimal norm to the resolution of the mixed formulation (24), or equivalently to the search of the saddle point for $\mathcal{L}$. In general, it is very convenient (and actually necessary in Section 3.2 to "augment" the Lagrangian (see [14]), and consider instead the Lagrangian $\mathcal{L}_{r}$ defined for any $r>0$ by

$$
\left\{\begin{array}{l}
\mathcal{L}_{r}(\varphi, \lambda):=\frac{1}{2} a_{r}(\varphi, \varphi)+b(\varphi, \lambda)-l(\varphi), \\
a_{r}(\varphi, \varphi):=a(\varphi, \varphi)+r\|L \varphi\|_{L^{2}\left(0, T ; H^{-1}(0,1)\right)}^{2}
\end{array}\right.
$$

Since $a(\varphi, \varphi)=a_{r}(\varphi, \varphi)$ on $W$, the Lagrangian $\mathcal{L}$ and $\mathcal{L}_{r}$ share the same saddle-point. 
Remark 2 The estimate (11) may also be used to extend the work [9] to the non-cylindrical situation. Reference [9] considers the pair $(y, v)$ solution of [1] 3) which minimize the following $L^{2}$-weighted functional

$$
J(y, v):=\frac{1}{2} \iint_{Q_{T}} \rho^{2}(x, t)|y|^{2} d x d t+\frac{1}{2} \iint_{q_{T}} \rho_{0}^{2}(x, t)|v|^{2} d x d t
$$

for any weights $\rho, \rho_{0} \in C\left(\overline{Q_{T}}, \mathbb{R}_{+}^{\star}\right)$. Assuming $|\rho|$ and $\left|\rho_{0}\right|$ uniformly positive by below, the unique minimizer $(y, v)$ is expressed in term of the auxiliary variable $p \in P:=\left\{p: \rho^{-1} L p \in\right.$ $L^{2}\left(Q_{T}\right), \rho_{0}^{-1} p \in L^{2}\left(q_{T}\right), p=0$ on $\left.\Sigma_{T}\right\}$ as follows :

$$
y=-\rho^{-2} L p, \quad v=\rho_{0}^{-2} p 1_{q_{T}} \quad \text { on } \quad Q_{T}
$$

where $p$ is the solution of the variational formulation

$$
\iint_{Q_{T}} \rho^{-2} L p L q d x d t+\iint_{q_{T}} \rho_{0}^{-2} p q d x d t=\int_{0}^{1} y^{1} q(\cdot, 0) d x-<y^{0}, q_{t}(\cdot, 0)>_{H_{0}^{1}(0,1), H^{-1}(0,1)}, \quad \forall q \in P .
$$

The well-posedness of this formulation is given by the estimate (11).

\subsection{Dual problem of the extremal problem (23)}

The mixed formulation allows to solve simultaneously the dual variable $\varphi$, argument of the conjugate functional (23), and the Lagrangian multiplier $\lambda$. Since $\lambda$ turns out to be the controlled state of (1), we may qualify $\lambda$ as the primal variable of the controllability problem. We derive in this section the corresponding extremal problem involving only that variable $\lambda$. by

For any $r>0$, let us define the linear operator $A_{r}$ from $L^{2}\left(0, T ; H_{0}^{1}(0,1)\right)$ into $L^{2}\left(0, T ; H_{0}^{1}(0,1)\right)$

$$
A_{r} \lambda:=-\Delta^{-1}(L \varphi), \quad \forall \lambda \in L^{2}\left(0, T ; H_{0}^{1}(0,1)\right)
$$

where $\varphi \in \Phi$ is the unique solution to

$$
a_{r}(\varphi, \bar{\varphi})=b(\bar{\varphi}, \lambda), \quad \forall \bar{\varphi} \in \Phi .
$$

Notice that the assumption $r>0$ is needed here in order to guarantee the well-posedness of 28]. Precisely, for any $r>0$, the form $a_{r}$ defines a norm equivalent to the norm on $\varphi$.

We have the following important lemma :

LEMma 3.1 For any $r>0$, the operator $A_{r}$ is a strongly elliptic, symmetric isomorphism from $L^{2}\left(0, T ; H_{0}^{1}(0,1)\right)$ into $L^{2}\left(0, T ; H_{0}^{1}(0,1)\right)$.

Proof- From the definition of $a_{r}$, we easily get that $\left\|A_{r} \lambda\right\|_{L^{2}\left(0, T ; H_{0}^{1}(0,1)\right)} \leq r^{-1}\|\lambda\|_{L^{2}\left(0, T ; H_{0}^{1}(0,1)\right)}$ and the continuity of $A_{r}$. Next, consider any $\lambda^{\prime} \in L^{2}\left(0, T ; H_{0}^{1}(0,1)\right)$ and denote by $\varphi^{\prime}$ the corresponding unique solution of 28) so that $A_{r} \lambda^{\prime}:=-\Delta^{-1}\left(L \varphi^{\prime}\right)$. Relation 28) with $\bar{\varphi}=\varphi^{\prime}$ then implies that

$$
\int_{0}^{T}<A_{r} \lambda^{\prime}, \lambda>_{H_{0}^{1}(0,1), H_{0}^{1}(0,1)} d t=a_{r}\left(\varphi, \varphi^{\prime}\right)
$$

and therefore the symmetry and positivity of $A_{r}$. The last relation with $\lambda^{\prime}=\lambda$ and the estimate (11) imply that $A_{r}$ is also positive definite.

Finally, let us check the strong ellipticity of $A_{r}$, equivalently that the bilinear functional $\left(\lambda, \lambda^{\prime}\right) \rightarrow \int_{0}^{T}<A_{r} \lambda, \lambda^{\prime}>_{H_{0}^{1}(0,1), H_{0}^{1}(0,1)} d t$ is $L^{2}\left(0, T ; H_{0}^{1}(0,1)\right)$-elliptic. Thus we want to show that

$$
\int_{0}^{T}<A_{r} \lambda, \lambda>_{H_{0}^{1}(0,1), H_{0}^{1}(0,1)} d t \geq C\|\lambda\|_{L^{2}\left(0, T ; H_{0}^{1}(0,1)\right)}^{2}, \quad \forall \lambda \in L^{2}\left(0, T ; H_{0}^{1}(0,1)\right)
$$


for some positive constant $C$. Suppose that (30) does not hold; there exists then a sequence $\left\{\lambda_{n}\right\}_{n \geq 0}$ of $L^{2}\left(0, T ; H_{0}^{1}(0,1)\right)$ such that

$$
\left\|\lambda_{n}\right\|_{L^{2}\left(0, T, H_{0}^{1}(0,1)\right)}=1, \quad \forall n \geq 0, \quad \text { and } \quad \lim _{n \rightarrow \infty} \int_{0}^{T}<A_{r} \lambda_{n}, \lambda_{n}>_{H_{0}^{1}(0,1), H_{0}^{1}(0,1)} d t=0 .
$$

Let us denote by $\varphi_{n}$ the solution of 28 corresponding to $\lambda_{n}$. From (29), we then obtain that

$$
\lim _{n \rightarrow \infty}\left\|L \varphi_{n}\right\|_{L^{2}\left(0, T, H^{-1}(0,1)\right)}=0, \quad \lim _{n \rightarrow+\infty}\left\|\varphi_{n}\right\|_{L^{2}\left(q_{T}\right)}=0
$$

and thus $\lim _{n \rightarrow \infty} \int_{0}^{T}<-\Delta^{-1}(L \varphi), \lambda_{n}>_{H_{0}^{1}(0,1), H_{0}^{1}(0,1)} d t=0$ for all $\varphi \in \Phi$ (and so the $L^{2}\left(0, T ; H_{0}^{1}(0,1)\right.$ )weak-convergence of $\lambda_{n}$ toward 0$)$.

From 28] with $\varphi=\varphi_{n}$ and $\lambda_{n}$, we have

$$
\int_{0}^{T}<-r \Delta^{-1}\left(L \varphi_{n}\right)-\lambda_{n},-\Delta^{-1}(L \bar{\varphi})>_{H_{0}^{1}(0,1), H_{0}^{1}(0,1)} d t+\iint_{q_{T}} \varphi_{n} \bar{\varphi} d x d t=0, \quad \forall \bar{\varphi} \in \Phi .
$$

We define the sequence $\left\{\bar{\varphi}_{n}\right\}_{n \geq 0}$ as follows :

$$
\left\{\begin{array}{l}
L \bar{\varphi}_{n}=r L \varphi_{n}+\Delta \lambda_{n}, \quad \text { in } \quad Q_{T}, \\
\bar{\varphi}_{n}(0, \cdot)=\bar{\varphi}_{n}(1, \cdot)=0, \quad \text { in } \quad(0, T), \\
\bar{\varphi}_{n}(\cdot, 0)=\bar{\varphi}_{n, t}(\cdot, 0)=0, \quad \text { in } \quad(0,1)
\end{array}\right.
$$

so that, for all $n, \bar{\varphi}_{n}$ is the solution of the wave equation with zero initial data and source term $r L \varphi_{n}+\Delta \lambda_{n}$ in $L^{2}\left(0, T ; H^{-1}(0,1)\right)$. Using again (27), we get $\left\|\bar{\varphi}_{n}\right\|_{L^{2}\left(q_{T}\right)} \leq C_{\Omega, T} \| r L \varphi_{n}+$ $\Delta \lambda_{n} \|_{L^{2}\left(0, T ; H^{-1}(0,1)\right)}$, so that $\bar{\varphi}_{n} \in \Phi$. Then, using (32), we get

$$
\left\|-r \Delta^{-1}\left(L \varphi_{n}\right)-\lambda_{n}\right\|_{L^{2}\left(0, T ; H_{0}^{1}(0,1)\right)} \leq C_{\Omega, T}\left\|\varphi_{n}\right\|_{L^{2}\left(q_{T}\right)} .
$$

Then, from (31), we conclude that $\lim _{n \rightarrow+\infty}\left\|\lambda_{n}\right\|_{L^{2}\left(0, T ; H_{0}^{1}(0,1)\right)}=0$ leading to a contradiction and to the strong ellipticity of the operator $A_{r}$.

The introduction of the operator $A_{r}$ is motivated by the following proposition :

Proposition 3.1 Let $\varphi_{0} \in \Phi$ the unique solution of

$$
a_{r}\left(\varphi_{0}, \bar{\varphi}\right)=l(\bar{\varphi}), \quad \forall \bar{\varphi} \in \Phi
$$

and let $J^{\star \star}: L^{2}\left(0, T ; H_{0}^{1}(0,1)\right) \rightarrow L^{2}\left(0, T ; H_{0}^{1}(0,1)\right)$ the functional defined by

$$
J^{\star \star}(\lambda)=\frac{1}{2} \int_{0}^{T}<A_{r} \lambda, \lambda>_{H_{0}^{1}(0,1), H_{0}^{1}(0,1)} d t-b\left(\varphi_{0}, \lambda\right) .
$$

The following equality holds :

$$
\sup _{\lambda \in L^{2}\left(0, T ; H_{0}^{1}(0,1)\right)} \inf _{\varphi \in \Phi} \mathcal{L}_{r}(\varphi, \lambda)=-\inf _{\lambda \in L^{2}\left(0, T ; H_{0}^{1}(0,1)\right)} J^{\star \star}(\lambda)+\mathcal{L}_{r}\left(\varphi_{0}, 0\right) .
$$

Proof- For any $\lambda \in L^{2}\left(0, T ; H_{0}^{1}(0,1)\right)$, let us denote by $\varphi_{\lambda} \in \Phi$ the minimizer of $\varphi \rightarrow \mathcal{L}_{r}(\varphi, \lambda)$. $\varphi_{\lambda}$ satisfies the equation

$$
a_{r}\left(\varphi_{\lambda}, \bar{\varphi}\right)+b(\bar{\varphi}, \lambda)=l(\bar{\varphi}), \quad \forall \bar{\varphi} \in \Phi
$$

and can be decomposed as follows : $\varphi_{\lambda}=\psi_{\lambda}+\varphi_{0}$ where $\psi_{\lambda} \in \Phi$ solves

$$
a_{r}\left(\psi_{\lambda}, \bar{\varphi}\right)+b(\bar{\varphi}, \lambda)=0, \quad \forall \bar{\varphi} \in \Phi .
$$


We then have

$$
\begin{aligned}
\inf _{\varphi \in \Phi} \mathcal{L}_{r}(\varphi, \lambda) & =\mathcal{L}_{r}\left(\varphi_{\lambda}, \lambda\right)=\mathcal{L}_{r}\left(\psi_{\lambda}+\varphi_{0}, \lambda\right) \\
& =\frac{1}{2} a_{r}\left(\psi_{\lambda}+\varphi_{0}, \psi_{\lambda}+\varphi_{0}\right)+b\left(\psi_{\lambda}+\varphi_{0}, \lambda\right)-l\left(\psi_{\lambda}+\varphi_{0}\right) \\
& :=X_{1}+X_{2}+X_{3}
\end{aligned}
$$

with

$$
\left\{\begin{array}{l}
X_{1}=\frac{1}{2} a_{r}\left(\psi_{\lambda}, \psi_{\lambda}\right)+b\left(\psi_{\lambda}, \lambda\right)+b\left(\varphi_{0}, \lambda\right) \\
X_{2}=a_{r}\left(\psi_{\lambda}, \varphi_{0}\right)-l\left(\psi_{\lambda}\right), \quad X_{3}=\frac{1}{2} a_{r}\left(\varphi_{0}, \varphi_{0}\right)-l\left(\varphi_{0}\right) .
\end{array}\right.
$$

From the definition of $\varphi_{0}, X_{2}=0$ while $X_{3}=\mathcal{L}_{r}\left(\varphi_{0}, 0\right)$. Eventually, from the definition of $\psi_{\lambda}$,

$$
X_{1}=-\frac{1}{2} a_{r}\left(\psi_{\lambda}, \psi_{\lambda}\right)+b\left(\varphi_{0}, \lambda\right)=-\frac{1}{2} \int_{0}^{T}<A_{r} \lambda, \lambda>_{H^{1}, H^{1}} d t+b\left(\varphi_{0}, \lambda\right)
$$

and the result follows.

From the ellipticity of the operator $A_{r}$, the minimization of the functional $J^{\star \star}$ over $L^{2}\left(0, T, H_{0}^{1}\right)$ is well-posed. It is interesting to note that with this extremal problem involving only $\lambda$, we are coming to the primal variable, controlled solution of (1) (see Theorem 3.1. (iii)). Due to the constraint (3), the direct minimization of the null controllability problem by a penalty method with respect to the controlled state is usually avoided in practice. Here, any constraint equality is assigned to the variable $\lambda$.

From the symmetry and ellipticity of the operator $A_{r}$, the conjugate gradient algorithm is very appropriate to minimize $J^{\star \star}$, and consequently solve the mixed formulation (24). The conjugate gradient algorithm reads as follows :

(i) Let $\lambda^{0} \in L^{2}\left(0, T ; H_{0}^{1}(0,1)\right)$ be a given function.

(ii) Compute $\bar{\varphi}^{0} \in \Phi$ solution to

$$
a_{r}\left(\bar{\varphi}^{0}, \bar{\varphi}\right)+b\left(\bar{\varphi}, \lambda^{0}\right)=l(\bar{\varphi}), \quad \forall \bar{\varphi} \in \Phi
$$

and $g^{0}=-\Delta^{-1}\left(L \bar{\varphi}^{0}\right)$ then set $w^{0}=g^{0}$.

(iii) For $n \geq 0$, assuming that $\lambda^{n}, g^{n}$ and $w^{n}$ are known, compute $\bar{\varphi}^{n} \in \Phi$ solution to

$$
a_{r}\left(\bar{\varphi}^{n}, \bar{\varphi}\right)=b\left(\bar{\varphi}, w^{n}\right), \quad \forall \bar{\varphi} \in \Phi
$$

and $\bar{g}^{n}=-\Delta^{-1}\left(L \bar{\varphi}^{n}\right)$ and then

$$
\rho_{n}=\left\|g^{n}\right\|_{L^{2}\left(0, T ; H_{0}^{1}(0,1)\right)}^{2} /\left(\bar{g}^{n}, w^{n}\right)_{L^{2}\left(0, T ; H_{0}^{1}(0,1)\right)} .
$$

Update $\lambda^{n}$ and $g^{n}$ by

$$
\lambda^{n+1}=\lambda^{n}-\rho_{n} w^{n}, \quad g^{n+1}=g^{n}-\rho^{n} \bar{g}^{n} .
$$

If $\left\|g^{n+1}\right\|_{L^{2}\left(0, T ; H_{0}^{1}(0,1)\right)} /\left\|g^{0}\right\|_{L^{2}\left(0, T ; H_{0}^{1}(0,1)\right)} \leq \varepsilon$, take $\lambda=\lambda^{n+1}$. Else, compute

$$
\gamma_{n}=\left\|g^{n+1}\right\|_{L^{2}\left(0, T ; H_{0}^{1}(0,1)\right)}^{2} /\left\|g^{n}\right\|_{L^{2}\left(0, T ; H_{0}^{1}(0,1)\right)}^{2}
$$

and update $w^{n}$ via

$$
w^{n+1}=g^{n+1}+\gamma_{n} w^{n} .
$$

Do $n=n+1$ and return to step (iii). 
As mentioned in [15] where this approach is discussed at length for Navier-Stokes type systems, this algorithm can be viewed as a sophisticated version of Arrow-Hurwicz-Uzawa type method.

Concerning the speed of convergence of the conjugate gradient algorithm (i)-(iii), it follows from for instance [12] that

$$
\left\|\lambda^{n}-\lambda\right\|_{L^{2}\left(0, T ; H_{0}^{1}(0,1)\right)} \leq 2 \sqrt{\nu\left(A_{r}\right)}\left(\frac{\sqrt{\nu\left(A_{r}\right)}-1}{\sqrt{\nu\left(A_{r}\right)}+1}\right)^{n}\left\|\lambda^{0}-\lambda\right\|_{L^{2}\left(0, T ; H_{0}^{1}(0,1)\right)}, \quad \forall n \geq 1
$$

where $\lambda$ minimizes $J^{\star \star} . \nu\left(A_{r}\right)=\left\|A_{r}\right\|\left\|A_{r}^{-1}\right\|$ denotes the condition number of the operator $A_{r}$.

Eventually, once the above algorithm has converged we can compute $\varphi \in \Phi$ as solution of

$$
a_{r}(\varphi, \bar{\varphi})+b(\bar{\varphi}, \lambda)=l(\bar{\varphi}), \quad \forall \bar{\varphi} \in \Phi
$$

\section{Numerical approximation and experiments}

\subsection{Some domains $q_{T}$}

Let us first describe the domains $q_{T} \subset Q_{T}:=(0,1) \times(0, T)$ in which the control is supported we shall use in our numerical experiments.

Let $\gamma_{T}^{i}:(0, T) \rightarrow(0,1)$ be four $C^{\infty}$ functions defined as follows :

$$
\begin{array}{rlrl}
\gamma_{T}^{0}(t) & =\frac{3}{10}, & t \in(0, T), \\
\gamma_{T}^{1}(t)=\frac{1}{2}+\frac{1}{10} \cos \left(\frac{\pi t}{T}\right), & t \in(0, T), \\
\gamma_{T}^{2}(t)=\frac{\beta-\alpha}{T} t+\alpha, & t \in(0, T), \\
\gamma_{T}^{3}(t)=\frac{1}{2}+\frac{1}{4} \cos \left(\frac{8 \pi t}{T}\right), & t \in(0, T) .
\end{array}
$$

In what follows we choose in (35) $\alpha=0.2$ and $\beta=0.8$.

For each $i \in\{0,1,2,3\}$, let $a_{T}^{i}, b_{T}^{i}:(0, T) \rightarrow(0,1)$ be two functions defined by

$$
a_{T}^{i}(t)=\gamma_{T}^{i}(t)-\delta_{0}, \quad b_{T}^{i}(t)=\gamma_{T}^{i}(t)+\delta_{0}, \quad t \in(0, T) .
$$

for some $\delta_{0}>0$ small enough. We then define the corresponding domains $q_{T}^{i}$ as follows :

$$
q_{T}^{i}=\left\{(x, t) \in Q_{T} ; a_{T}^{i}(t)<x<b_{T}^{i}(t), t \in(0, T)\right\}, \quad i \in\{0,1,2,3\} .
$$

Remark that, in the definition of $a_{T}^{i}$ and $b_{T}^{i}$, we may consider time-dependent value for $\delta_{0}$. Figure 2 display the domains $q_{T}^{i}$ defined by (38) with the controllability time $T=2.2$ and $\delta_{0}=10^{-1}$. We easily check, that for any $T>2$, these domains satisfy the geometric condition of Proposition 2.1.

\subsection{Discretization}

We now turn to the discretization of the mixed formulation (24) assuming $r>0$.

Let then $\Phi_{h}$ and $M_{h}$ be two finite dimensional spaces parametrized by the variable $h$ such that

$$
\Phi_{h} \subset \Phi, \quad M_{h} \subset L^{2}\left(0, T ; H_{0}^{1}(0,1)\right), \quad \forall h>0 .
$$

Then, we can introduce the following approximated problems : find $\left(\varphi_{h}, \lambda_{h}\right) \in \Phi_{h} \times M_{h}$ solution of

$$
\left\{\begin{aligned}
a_{r}\left(\varphi_{h}, \bar{\varphi}_{h}\right)+b\left(\bar{\varphi}_{h}, \lambda_{h}\right) & =l\left(\bar{\varphi}_{h}\right), & & \forall \bar{\varphi}_{h} \in \Phi_{h} \\
b\left(\varphi_{h}, \bar{\lambda}_{h}\right) & =0, & & \forall \bar{\lambda}_{h} \in M_{h} .
\end{aligned}\right.
$$



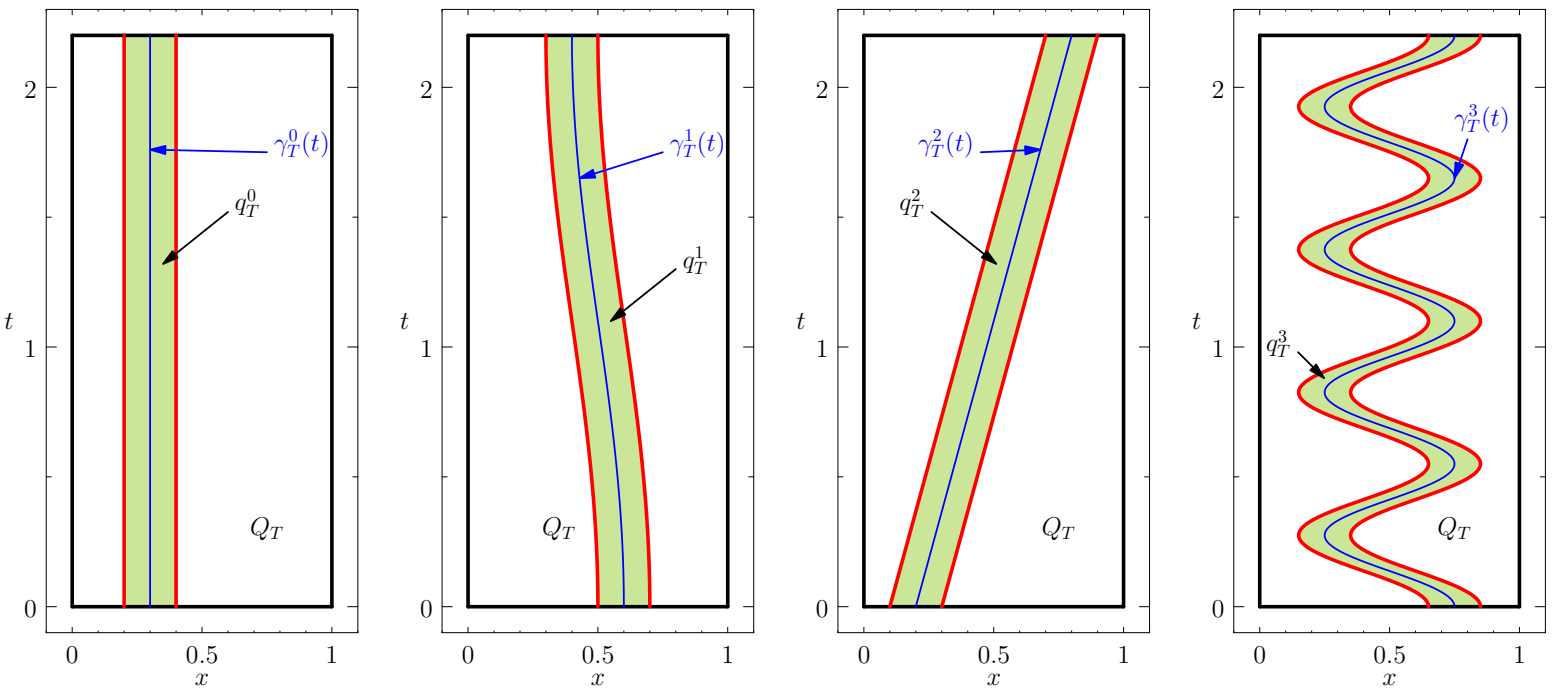

Figure 2: The time dependent domains $q_{T}^{i}, i \in\{0,1,2,3\}$ defined by 38.

The well-posedness of this mixed formulation is again a consequence of two properties : the coercivity of the bilinear form $a_{r}$ on the subset $\mathcal{N}_{h}(b)=\left\{\varphi_{h} \in \Phi_{h} ; b\left(\varphi_{h}, \lambda_{h}\right)=0 \quad \forall \lambda_{h} \in M_{h}\right\}$. Actually, from the relation

$$
a_{r}(\varphi, \varphi) \geq \frac{r}{\eta}\|\varphi\|_{\Phi}^{2}, \quad \forall \varphi \in \Phi
$$

the form $a_{r}$ is coercive on the full space $\Phi$, and so a fortiori on $\mathcal{N}_{h}(b) \subset \Phi_{h} \subset \Phi$. The second property is a discrete inf-sup condition : there exists $\delta_{h}>0$ such that

$$
\inf _{\lambda_{h} \in M_{h}} \sup _{\varphi_{h} \in \Phi_{h}} \frac{b\left(\varphi_{h}, \lambda_{h}\right)}{\left\|\varphi_{h}\right\|_{\Phi_{h}}\left\|\lambda_{h}\right\|_{M_{h}}} \geq \delta_{h} .
$$

For any fixed $h$, the spaces $M_{h}$ and $\Phi_{h}$ are of finite dimension so that the infimum and supremum in (40) are reached: moreover, from the property of the bilinear form $a_{r}$, it is standard to prove that $\delta_{h}$ is strictly positive (see Section 4.5). Consequently, for any fixed $h>0$, there exists a unique couple $\left(\varphi_{h}, \lambda_{h}\right)$ solution of (39). On the other hand, the property $\inf _{h} \delta_{h}>0$ is in general difficult to prove and depends strongly on the choice made for the approximated spaces $M_{h}$ and $\Phi_{h}$. We shall analyze numerically this property in Section 4.5 .

The finite dimensional and conformal space $\Phi_{h}$ must be chosen such that $L \varphi_{h}$ belongs to $L^{2}\left(0, T, H^{-1}(0,1)\right)$ for any $\varphi_{h} \in \Phi_{h}$. This is guaranteed for instance as soon as $\varphi_{h}$ possesses second-order derivatives in $L_{l o c}^{2}\left(Q_{T}\right)$. Therefore, a conformal approximation based on standard triangulation of $Q_{T}$ requires spaces of functions continuously differentiable with respect to both variables $x$ and $t$.

We introduce a triangulation $\mathcal{T}_{h}$ such that $\overline{Q_{T}}=\cup_{K \in \mathcal{T}_{h}} K$ and we assume that $\left\{\mathcal{T}_{h}\right\}_{h>0}$ is a regular family. We note

$$
h:=\max \left\{\operatorname{diam}(K), K \in \mathcal{T}_{h}\right\}
$$

where $\operatorname{diam}(K)$ denotes the diameter of $K$. Then, we introduce the space $\Phi_{h}$ as follows :

$$
\Phi_{h}=\left\{\varphi_{h} \in \Phi_{h} \in C^{1}\left(\overline{Q_{T}}\right):\left.\varphi_{h}\right|_{K} \in \mathbb{P}(K) \quad \forall K \in \mathcal{T}_{h}, \varphi_{h}=0 \text { on } \Sigma_{T}\right\}
$$

where $\mathbb{P}(K)$ denotes an appropriate space of polynomial functions in $x$ and $t$. In this work, we consider for $\mathbb{P}(K)$ the reduced Hsieh-Clough-Tocher (HCT for short) $C^{1}$-element. This is a so-called composite finite element and involves 9 degrees of freedom, namely the values of $\varphi_{h}, \varphi_{h, x}, \varphi_{h, t}$ on the 
three vertices of each triangle $K$. We refer to [8] page 356 and to [2, 21] where the implementation is discussed.

We also define the finite dimensional space

$$
M_{h}=\left\{\lambda_{h} \in C^{0}\left(\overline{Q_{T}}\right),\left.\lambda_{h}\right|_{K} \in \mathbb{Q}(K) \quad \forall K \in \mathcal{T}_{h}, \lambda_{h}=0 \text { on } \Sigma_{T}\right\}
$$

where $\mathbb{Q}(K)$ denotes the space of affine functions both in $x$ and $t$ on the element $K$. For any $h>0$, we have $\Phi_{h} \subset \Phi$ and $M_{h} \subset L^{2}\left(0, T ; H_{0}^{1}(0,1)\right)$.

For each combination of domains $\left(q_{T}, Q_{T}\right)$ described in Section 4.1 we consider six levels of triangulations $\mathcal{T}_{h}$ (numbered from $\sharp 0$ to $\sharp 5$, from coarser to finer). The number of triangles for some examples of domains $q_{T}$ which will be used the experiments are summarized in Table 1. In Figure 3 we display the meshes $\sharp 1$ corresponding to geometries described in Figure 2 .

\begin{tabular}{|c|cccccc|}
\hline$\sharp$ Mesh & 0 & 1 & 2 & 3 & 4 & 5 \\
\hline$q_{T=2.2}^{0}$ & 207 & 828 & 3312 & 13248 & 52992 & 211968 \\
$q_{T=2}^{0}$ & 198 & 792 & 3168 & 12672 & 50688 & 202752 \\
$q_{T=2.2}^{1}$ & 150 & 600 & 2400 & 9600 & 38400 & 153600 \\
$q_{T=2.2}^{2}$ & 179 & 716 & 2864 & 11456 & 45824 & 183296 \\
$q_{T=2}^{2}$ & 177 & 708 & 2832 & 11328 & 45312 & 181248 \\
$q_{T=2.2}^{3}$ & 464 & 1856 & 7424 & 29696 & 118784 & 475136 \\
\hline
\end{tabular}

Table 1: Number of triangles for different meshes and different control domains $q_{T}^{i}$
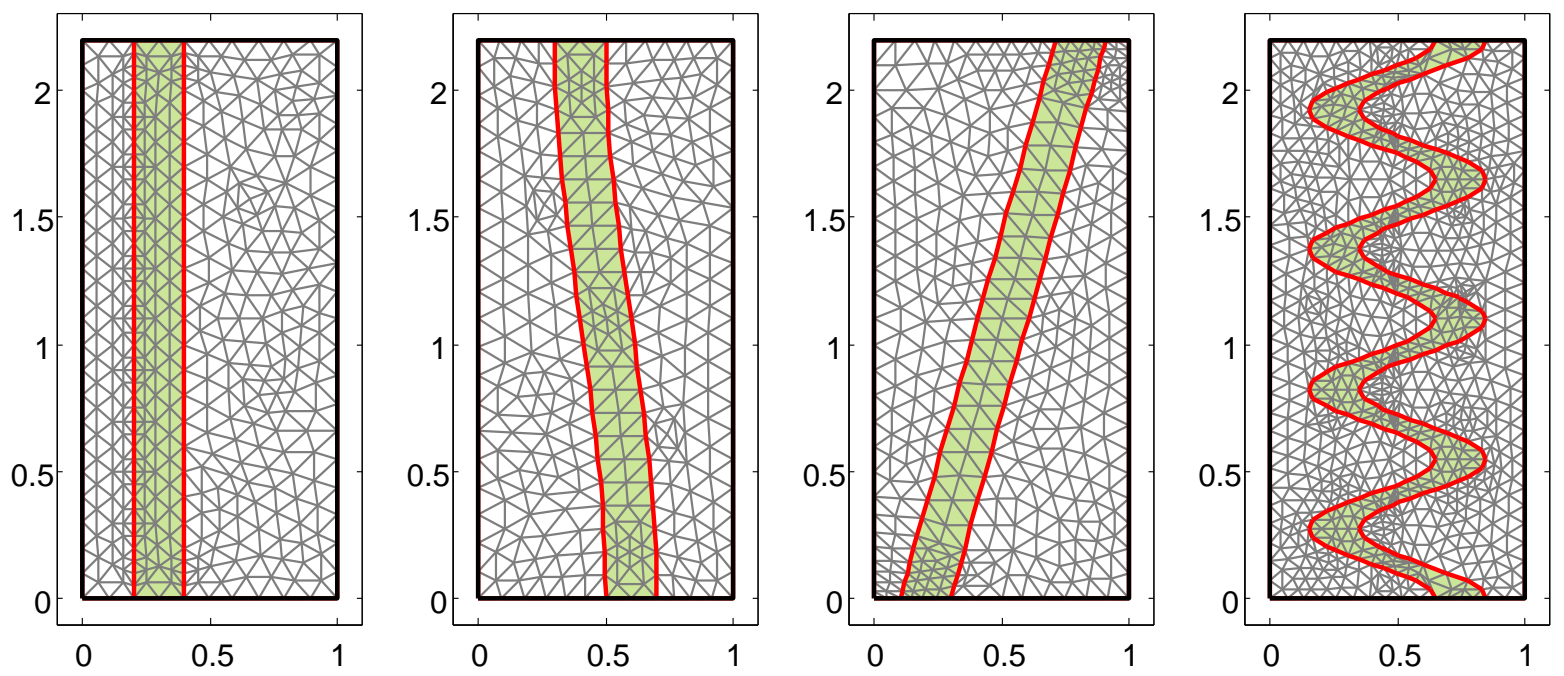

Figure 3: Meshes $\sharp 1$ associated with the domains $q_{T=2.2}^{i}: i=0,1,2,3$ from left to right.

\subsection{Change of the norm $\|\cdot\|_{L^{2}\left(H^{-1}\right)}$ over the discrete space $\Phi_{h}$}

In contrast to [10] where the boundary controllability is considered with the constraint $L \varphi=0$ as an $L^{2}\left(Q_{T}\right)$ function, the equality $L \varphi=0$ in $\Phi$ is assumed in the weaker space $L^{2}\left(0, T ; H^{-1}(0,1)\right)$. It is not straightforward to handle numerically the scalar product over $H^{-1}$ which appears in the mixed formulation (39). However, at the finite dimensional level of the discretization, since all the norms are equivalent, a classical trick (see for instance 3, 4]) consists in replacing, for any fixed $h$, the norm $\left\|L \varphi_{h}\right\|_{L^{2}\left(0, T ; H^{-1}(0,1)\right)}$ by the norm $\left\|L \varphi_{h}\right\|_{L^{2}\left(Q_{T}\right)}$, up to a constant. 
In order to do that, first remark that if there exist two constants $C_{0}>0$ and $\alpha>0$ such that

$$
\left\|\psi_{h}\right\|_{L^{2}\left(Q_{T}\right)}^{2} \geq C_{0} h^{\alpha}\left\|\psi_{h}\right\|_{L^{2}\left(0, T ; H_{0}^{1}(0,1)\right)}^{2}, \quad \forall \psi_{h} \in \Phi_{h}
$$

then a similar inequality it holds for weaker norms. More precisely, we have

$$
\left\|\varphi_{h}\right\|_{L^{2}\left(0, T ; H^{-1}(0,1)\right)}^{2} \geq C_{0} h^{\alpha}\left\|\varphi_{h}\right\|_{L^{2}\left(Q_{T}\right)}^{2}, \quad \forall \varphi_{h} \in \Phi_{h} .
$$

Indeed, to obtain 42 it suffices to take $\psi_{h}(\cdot, t)=(-\Delta)^{\frac{1}{2}} \varphi_{h}(\cdot, t)$ in 41]. That gives

$$
\int_{0}^{T}\left\|(-\Delta)^{-\frac{1}{2}} \varphi_{h}(\cdot, t)\right\|_{L^{2}(0,1)}^{2} d t \geq C_{0} h^{\alpha} \int_{0}^{T}\left\|(-\Delta)^{-\frac{1}{2}} \varphi_{h, x}(\cdot, t)\right\|_{L^{2}(0,1)}^{2} d t .
$$

Since $-\Delta$ is a self-adjoint positive operator and $\varphi_{h} \in \Phi_{h} \subset H_{0}^{1}\left(Q_{T}\right)$ we can integrate by parts in both hand-sides of the above inequality and hence we deduce estimate 422 . We highlight that the term $C_{0} h^{\alpha}$ (and so $C_{0}$ and $\alpha$ ) does not depend on $T$.

Assuming that (41) (and consequently (42) holds (the constants $C_{0}, \alpha>0$ will be approximated numerically in Section 4.4, we may consider, for any fixed $h>0$, the following equivalent definitions of the form $a_{r, h}$ and $b_{h}$ over the finite dimensional spaces $\Phi_{h} \times \Phi_{h}$ and $\Phi_{h} \times M_{h}$ respectively :

$$
\begin{aligned}
a_{r, h}: \Phi_{h} \times \Phi_{h} \rightarrow \mathbb{R}, & a_{r, h}\left(\varphi_{h}, \overline{\varphi_{h}}\right)=a\left(\varphi_{h}, \overline{\varphi_{h}}\right)+r C_{0} h^{\alpha} \iint_{Q_{T}} L \varphi_{h} L \overline{\varphi_{h}} d x d t \\
b_{h}: \Phi_{h} \times M_{h} \rightarrow \mathbb{R}, & b_{h}\left(\varphi_{h}, \lambda_{h}\right)=\iint_{Q_{T}} L \varphi_{h} \lambda_{h} d x d t .
\end{aligned}
$$

Let $n_{h}=\operatorname{dim} \Phi_{h}, m_{h}=\operatorname{dim} M_{h}$ and let the real matrices $A_{r, h} \in \mathbb{R}^{n_{h}, n_{h}}, B_{h} \in \mathbb{R}^{m_{h}, n_{h}}$, $J_{h} \in \mathbb{R}^{m_{h}, m_{h}}$ and $L_{h} \in \mathbb{R}^{n_{h}, 1}$ be defined by

$$
\begin{array}{ll}
a_{r, h}\left(\varphi_{h}, \overline{\varphi_{h}}\right)=\left\langle A_{r, h}\left\{\varphi_{h}\right\},\left\{\overline{\varphi_{h}}\right\}\right\rangle_{\mathbb{R}^{n_{h}, \mathbb{R}^{n_{h}}}}, & \forall \varphi_{h}, \overline{\varphi_{h}} \in \Phi_{h}, \\
b_{h}\left(\varphi_{h}, \lambda_{h}\right)=\left\langle B_{h}\left\{\varphi_{h}\right\},\left\{\lambda_{h}\right\}\right\rangle_{\mathbb{R}^{m_{h}, \mathbb{R}^{m_{h}}},}, & \forall \varphi_{h} \in \Phi_{h}, \forall \lambda_{h} \in M_{h}, \\
\iint_{Q_{T}} \lambda_{h} \overline{\lambda_{h}} d x d t=\left\langle J_{h}\left\{\lambda_{h}\right\},\left\{\overline{\lambda_{h}}\right\}\right\rangle_{\mathbb{R}^{m_{h}, \mathbb{R}^{m_{h}}},}, \overline{\lambda_{h}}, \overline{\lambda_{h}} \in M_{h}, \\
l\left(\varphi_{h}\right)=\left\langle L_{h},\left\{\varphi_{h}\right\}\right\rangle, & \forall \varphi_{h} \in \Phi_{h}
\end{array}
$$

where $\left\{\varphi_{h}\right\} \in \mathbb{R}^{n_{h}, 1}$ denotes the vector associated to $\varphi_{h}$ and $\langle\cdot, \cdot\rangle_{\mathbb{R}^{n_{h}}, \mathbb{R}^{n_{h}}}$ the usual scalar product over $\mathbb{R}^{n_{h}}$. With these notations, the problem 39 reads as follows : find $\left\{\varphi_{h}\right\} \in \mathbb{R}^{n_{h}, 1}$ and $\left\{\lambda_{h}\right\} \in \mathbb{R}^{m_{h}, 1}$ such that

$$
\left(\begin{array}{cc}
A_{r, h} & B_{h}^{T} \\
B_{h} & 0
\end{array}\right)_{\mathbb{R}^{n_{h}+m_{h}, n_{h}+m_{h}}}\left(\begin{array}{c}
\left\{\varphi_{h}\right\} \\
\left\{\lambda_{h}\right\}
\end{array}\right)_{\mathbb{R}^{n_{h}+m_{h}, 1}}=\left(\begin{array}{c}
L_{h} \\
0
\end{array}\right)_{\mathbb{R}^{n_{h}+m_{h}, 1}} .
$$

The matrix $A_{r, h}$ as well as the mass matrix $J_{h}$ are symmetric and positive definite for any $h>0$ and any $r>0$. On the other hand, the main matrix of order $m_{h}+n_{h}$ in 49 is symmetric but not positive definite. We use exact integration methods developed in 13. for the evaluation of the coefficients of the matrices. The system $\sqrt{49}$ is solved using the direct LU decomposition method.

Let us also mention that for $r=0$, although the formulation (24) is well-posed, numerically, the corresponding matrix $A_{0, h}$ is not invertible. In the sequel, we shall consider strictly positive values for $r$.

Once the approximation $\varphi_{h}$ is obtained, an approximation $v_{h}$ of the control $v$ is given by $v_{h}=-\varphi_{h} 1_{q_{T}} \in L^{2}\left(Q_{T}\right)$. The corresponding controlled state $y_{h}$ may be obtained by solving (1) with standard forward approximation (we refer to $[9$, Section 4 where this is detailed). Here, since the controlled state is directly given by the multiplier $\lambda$, we simply use $\lambda_{h}$ as an approximation of $y$ and we do not report here the computation of $y_{h}$. 


\subsection{Numerical approximation of $C_{0}$ and $\alpha$ in (42).}

In order to approximate the values of the constants $C_{0}, \alpha$ appearing in 417 - 42 we consider the following problem :

$$
\text { find } \alpha>0 \text { and } C_{0}>0 \text { such that } \sup _{\varphi_{h} \in \Phi_{h}} \frac{\left\|\varphi_{h}\right\|_{L^{2}\left(0, T ; H_{0}^{1}(0,1)\right)}^{2}}{\left\|\varphi_{h}\right\|_{L^{2}\left(Q_{T}\right)}^{2}} \leq \frac{1}{C_{0} h^{\alpha}}, \quad \forall h>0 .
$$

Since $\Phi_{h}$ is a finite dimensional space, the supremum is, for any fixed $h>0$, the solution of the following eigenvalue problem :

$$
\forall h>0, \quad \gamma_{h}=\sup \left\{\gamma: K_{h}\left\{\psi_{h}\right\}=\gamma \bar{J}_{h}\left\{\psi_{h}\right\}, \quad \forall\left\{\psi_{h}\right\} \in \mathbb{R}^{m_{h}} \backslash\{0\}\right\}
$$

where $K_{h} \in \mathbb{R}^{n_{h}, n_{h}}$ and $\bar{J}_{h} \in \mathbb{R}^{n_{h}, n_{h}}$ are the matrices defined by

$$
\begin{array}{ll}
\left\langle K_{h}\left\{\psi_{h}\right\},\left\{\bar{\psi}_{h}\right\}\right\rangle_{\mathbb{R}^{n_{h}, \mathbb{R}^{n_{h}}}}=\iint_{Q_{T}} \psi_{h, x} \bar{\psi}_{h, x} d x d t, & \forall \psi_{h}, \bar{\psi}_{h} \in \Phi_{h}, \\
\left\langle\bar{J}_{h}\left\{\psi_{h}\right\},\left\{\bar{\psi}_{h}\right\}\right\rangle_{\mathbb{R}^{n_{h}, \mathbb{R}^{n_{h}}}}=\iint_{Q_{T}} \psi_{h} \bar{\psi}_{h} d x d t, & \forall \psi_{h}, \bar{\psi}_{h} \in \Phi_{h} .
\end{array}
$$

We then can choose $C_{0}$ and $\alpha$ in (50) such that $C_{0} h^{\alpha}=\gamma_{h}^{-1}$, where $\gamma_{h}$ solves the problem (51). Figure 4 displays $\gamma_{h}^{-1}$ corresponding to the matrices $K_{h}$ and $\bar{J}_{h}$ associated to the domains $Q_{T}$ and $q_{T}^{0}$ for the six levels of mesh and $T=2.2$. The values of constants $C_{0}$ and $\alpha$ which provide the best fitting are $C_{0} \approx 1.48 \times 10^{-2}$ and $\alpha=2.1993$. As expected, we also check that the constant $\gamma_{h}$ (and so $C_{0}$ and $\alpha$ ) does not depend on $T$ nor on the controllability domain. From now on, we use these numerical values in the bilinear form $a_{r, h}$ defined by 43 .

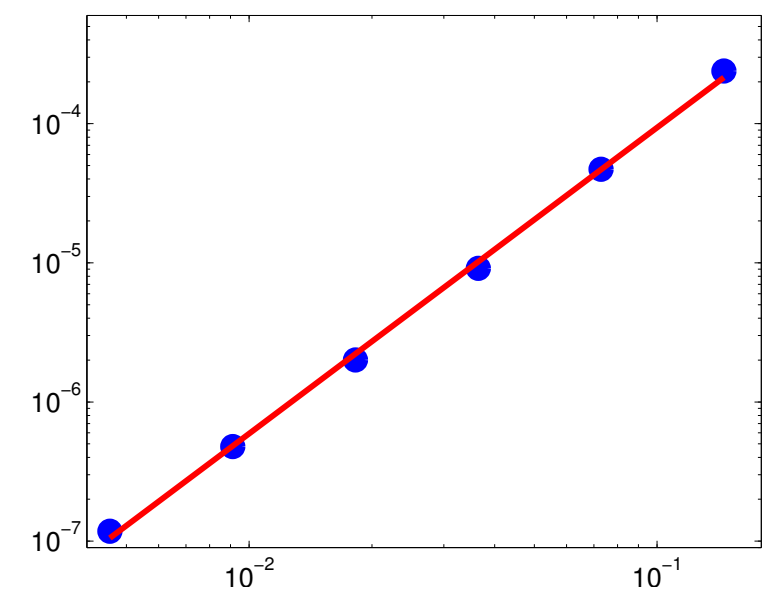

Figure 4: Values of $\gamma_{h}^{-1}$ vs. $h(\bullet)$. The line represents $C_{0} h^{\alpha}$ for $C_{0} \approx 1.48 \times 10^{-2}$ and $\alpha \approx 2.1993$.

\subsection{The discrete inf-sup test}

In order to solve the mixed formulation (39), we first test numerically the discrete inf-sup condition (40). Taking $\eta=r>0$ in 10 so that $a_{r, h}(\varphi, \bar{\varphi})=(\varphi, \bar{\varphi})_{\Phi}$ for all $\varphi, \bar{\varphi} \in \Phi$, it is readily seen (see for instance [7]) that the discrete inf-sup constant satisfies

$$
\delta_{h}:=\inf \left\{\sqrt{\delta}: B_{h} A_{r, h}^{-1} B_{h}^{T}\left\{\lambda_{h}\right\}=\delta J_{h}\left\{\lambda_{h}\right\}, \quad \forall\left\{\lambda_{h}\right\} \in \mathbb{R}^{m_{h}} \backslash\{0\}\right\} .
$$


As in the case of boundary controls (see [10]), the matrix $B_{h} A_{r, h}^{-1} B_{h}^{T}$ is symmetric and positive definite so that the real $\delta_{h}$ defined in term of the (generalized) eigenvalue problem (52) is, for any fixed value of the discretization parameter $h$, strictly positive. This eigenvalue problem is solved using the power iteration algorithm (assuming that the lowest eigenvalue is simple): for any $\left\{v_{h}^{0}\right\} \in \mathbb{R}^{n_{h}}$ such that $\left\|\left\{v_{h}^{0}\right\}\right\|_{2}=1$, compute for any $n \geq 0,\left\{\varphi_{h}^{n}\right\} \in \mathbb{R}^{n_{h}},\left\{\lambda_{h}^{n}\right\} \in \mathbb{R}^{m_{h}}$ and $\left\{v_{h}^{n+1}\right\} \in \mathbb{R}^{m_{h}}$ iteratively as follows :

$$
\left\{\begin{array}{l}
A_{r, h}\left\{\varphi_{h}^{n}\right\}+B_{h}^{T}\left\{\lambda_{h}^{n}\right\}=0 \\
B_{h}\left\{\varphi_{h}^{n}\right\}=-J_{h}\left\{v_{h}^{n}\right\}
\end{array} \quad, \quad\left\{v_{h}^{n+1}\right\}=\frac{\left\{\lambda_{h}^{n}\right\}}{\left\|\left\{\lambda_{h}^{n}\right\}\right\|_{2}} .\right.
$$

The scalar $\delta_{h}$ defined by 52 is then given by : $\delta_{h}=\lim _{n \rightarrow \infty}\left(\left\|\left\{\lambda_{h}^{n}\right\}\right\|_{2}\right)^{-1 / 2}$.

Table 2 reports the values of $\delta_{h}$ for various mesh sizes $h$, for $r=10^{-1}$ and $r=10^{-3}$ and for $q_{T}=q_{2.2}^{2}$. As expected, we check that $\delta_{h}$ decreases as $h \rightarrow 0$ and increases as $r \rightarrow 0$. More importantly, this table suggests that the sequence $\delta_{h}$ remains uniformly bounded by below with respect to $h$. This property remains true for other control domains $q_{T}$, as emphasized by Figure 5 .

\begin{tabular}{|c|ccccc|}
\hline$\sharp$ Mesh & 1 & 2 & 3 & 4 & 5 \\
\hline$h$ & $7.18 \times 10^{-2}$ & $3.59 \times 10^{-2}$ & $1.79 \times 10^{-2}$ & $8.97 \times 10^{-3}$ & $4.49 \times 10^{-3}$ \\
$r=10^{-1}$ & 18.8171 & 17.5466 & 17.0642 & 16.8880 & 16.8254 \\
$r=10^{3}$ & 0.6981 & 0.8374 & 0.9246 & 0.9964 & 1.0826 \\
\hline
\end{tabular}

Table 2: $\delta_{h}$ vs. $h$ for $q_{T}=q_{2}^{2}, r=10^{-1}$ and $r=10^{3}$.

We may conclude that the finite elements we use do "pass" the discrete inf-sup test. As we shall see in the next section, this fact implies the convergence of the sequence $\varphi_{h}$ and $\lambda_{h}$.

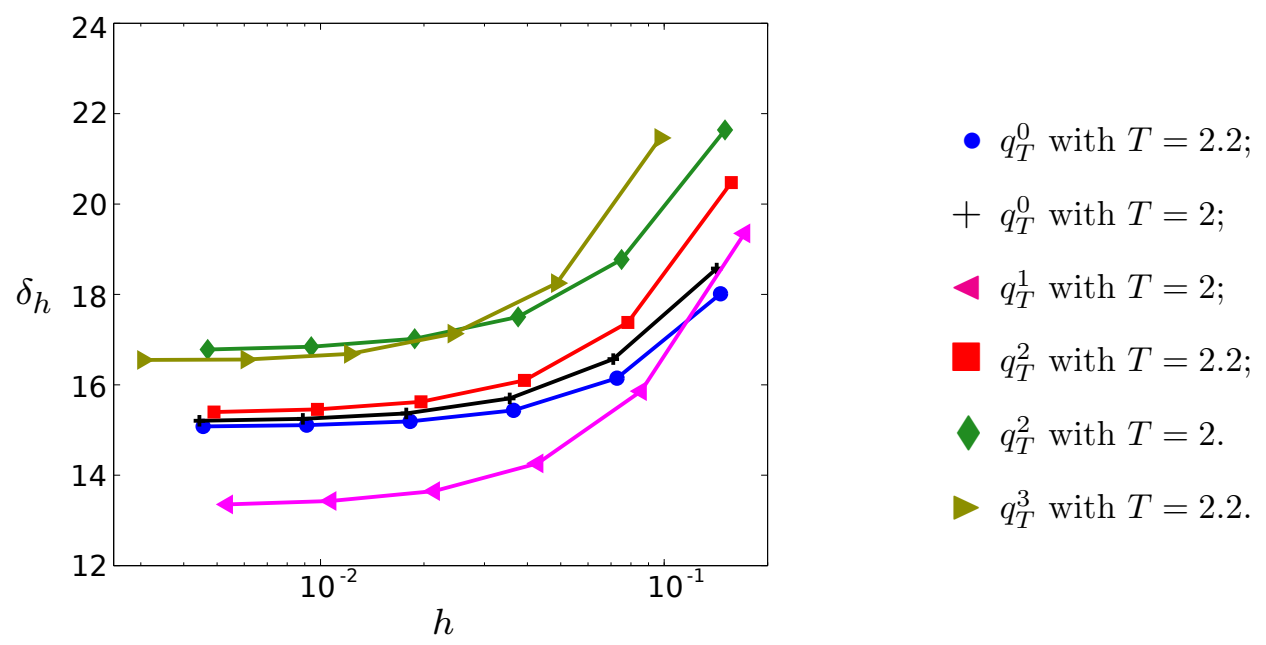

Figure 5: Values of $\delta_{h}$ vs. $h$ for different control domains $q_{T}^{i}$ and $r=10^{-1}$. 


\subsection{Numerical experiments for $q_{T}=q_{2}^{2}$ and comparison with the explicit solution}

We first consider the domain $q_{T}=q_{2}^{2}$ (see Figure 2) corresponding to an oblique band of length $2 \delta_{0}=0.2$ and $T=2$. We define also the following three initial data in $\boldsymbol{V}:=H_{0}^{1}(0,1) \times L^{2}(0,1)$ :

$$
\begin{array}{ll}
(\text { EX1 }) & y_{0}(x)=\sin (\pi x), \quad y_{1}(x)=0, \quad x \in(0,1), \\
(\text { EX2) } & y_{0}(x)=e^{-500(x-0.8)^{2}}, \quad y_{1}(x)=0, \quad x \in(0,1), \\
(\text { EX3 }) & y_{0}(x)=\frac{x}{\theta} 1_{(0, \theta)}(x)+\frac{1-x}{1-\theta} 1_{(\theta, 1)}(x), \quad y_{1}(x)=0, \quad \theta \in(0,1), \quad x \in(0,1) .
\end{array}
$$

In the case where the domain $q_{T}$ depends on the variable $t$, there is no in general exact solution of the mixed formulation (24. However, we can obtain a semi-explicit representation (using Fourier decomposition) of the minimizer $\left(\varphi_{0}, \varphi_{1}\right)$ of the conjugate functional $J^{\star}$ (see (8)), and consequently of the corresponding adjoint variable $\varphi$, the control of minimal square integrable norm $v=-\varphi 1_{q_{T}}$ and finally the controlled state $y$ solution of $(1-3)$. In practice, the obtention of the Fourier representation amounts to solve a symmetric linear system. We refer to the Appendix for the details. This allows to evaluate precisely the error $\left\|v-v_{h}\right\|_{L^{2}\left(q_{T}\right)}$ with respect to $h$ and confirm the relevance of the method.

Table 3 and 4 collects some numerical values for $r=10^{-1}$ and $r=10^{3}$ respectively corresponding to the initial data (EX1). In the Tables, $\kappa$ denotes the condition number associated to the linear system (49), independent of the initial data $\left(y_{0}, y_{1}\right)$. The convergence of $\left\|v-v_{h}\right\|_{L^{2}\left(q_{T}\right)}$, $\left\|L \varphi_{h}\right\|_{L^{2}\left(0, T ; H^{-1}(0,1)\right)}$ and $\left\|y-\lambda_{h}\right\|_{L^{2}\left(q_{T}\right)}$ toward zero as $h \searrow 0$ is clearly observed. This is fully in agreement with the uniform discrete inf-sup property we have observed in Section 4.5. We obtain the following rates of convergence with respect to $h$ for $r=10^{-1}$ and $r=10^{3}$ respectively :

$$
\begin{aligned}
& r=10^{-1}:\left\|v-v_{h}\right\|_{L^{2}\left(q_{T}\right)} \approx O\left(h^{1.3}\right),\left\|L \varphi_{h}\right\|_{L^{2}\left(0, T ; H^{-1}(0,1)\right)} \approx O\left(h^{1.3}\right),\left\|y-\lambda_{h}\right\|_{L^{2}\left(Q_{T}\right)} \approx O\left(h^{1.94}\right) \\
& r=10^{3}:\left\|v-v_{h}\right\|_{L^{2}\left(q_{T}\right)} \approx O\left(h^{1.09}\right), \quad\left\|L \varphi_{h}\right\|_{L^{2}\left(Q_{T}\right)} \approx O\left(h^{1.04}\right), \quad\left\|y-\lambda_{h}\right\|_{L^{2}\left(Q_{T}\right)} \approx O\left(h^{2.01}\right) .
\end{aligned}
$$

We refer to Figure 6 which highlights for $r=10^{-1}$ the polynomial convergence of the sequences $\left\|y-\lambda_{h}\right\|_{L^{2}\left(Q_{T}\right)}(" \vee)$ and $\left\|v-v_{h}\right\|_{L^{2}\left(q_{T}^{2}\right)}(" \bullet ")$ with respect to $h$. The previous rates suggests that the value of the parameter $r$ has a restricted influence.

\begin{tabular}{|c|ccccc|}
\hline$\sharp$ Mesh & 1 & 2 & 3 & 4 & 5 \\
\hline$h$ & $7.18 \times 10^{-2}$ & $3.59 \times 10^{-2}$ & $1.79 \times 10^{-2}$ & $8.97 \times 10^{-3}$ & $4.49 \times 10^{-3}$ \\
$\left\|v_{h}\right\|_{L^{2}\left(q_{T}\right)}$ & 5.370 & 5.047 & 4.893 & 4.815 & 4.776 \\
$\left\|L \varphi_{h}\right\|_{L^{2}\left(0, T ; H^{-1}(0,1)\right)}$ & 2.286 & $9.43 \times 10^{-1}$ & $3.76 \times 10^{-1}$ & $1.5 \times 10^{-1}$ & $6.15 \times 10^{-2}$ \\
$\left\|v-v_{h}\right\|_{L^{2}\left(q_{T}\right)}$ & $2.45 \times 10^{-1}$ & $9.65 \times 10^{-2}$ & $4.32 \times 10^{-2}$ & $2.29 \times 10^{-2}$ & $1.10 \times 10^{-2}$ \\
$\left\|y-\lambda_{h}\right\|_{L^{2}\left(Q_{T}\right)}$ & $5.63 \times 10^{-3}$ & $1.57 \times 10^{-3}$ & $4.04 \times 10^{-4}$ & $1.03 \times 10^{-4}$ & $2.61 \times 10^{-5}$ \\
$\kappa$ & $2.46 \times 10^{7}$ & $2.67 \times 10^{8}$ & $2.96 \times 10^{9}$ & $3.03 \times 10^{10}$ & $3.08 \times 10^{11}$ \\
\hline
\end{tabular}

Table 3: Example EX1; $q_{T}=q_{2}^{2} ; r=10^{-1}$.

The convergence of the method is also observed for the initial data (EX2), mainly supported around $x=0.8$ and the less regular data (EX3). Table 5 collects numerical values associated to (EX2), $q_{T}=q_{2}^{2}$ and $r=10^{-1}$. We obtain the following rates :

$$
\left\|v-v_{h}\right\|_{L^{2}\left(q_{T}\right)} \approx e^{5.85} h^{1.4},\left\|L \varphi_{h}\right\|_{L^{2}\left(Q_{T}\right)} \approx e^{7.96} h^{1.31},\left\|y-\lambda_{h}\right\|_{L^{2}\left(Q_{T}\right)} \approx e^{1.508} h^{1.62}
$$

Figure 7 displays other $Q_{T}$ the dual variable $\varphi_{h}$ and the primal variable $\lambda_{h}$ for $q_{T}=q_{2.2}^{2}$. The figures are obtained with the mesh $\sharp 3$. As expected, theses variable are mainly concentrated along the characteristics starting from $x=0.8$. 


\begin{tabular}{|c|ccccc|}
\hline$\sharp$ Mesh & 1 & 2 & 3 & 4 & 5 \\
\hline$h$ & $7.18 \times 10^{-2}$ & $3.59 \times 10^{-2}$ & $1.79 \times 10^{-2}$ & $8.97 \times 10^{-3}$ & $4.49 \times 10^{-3}$ \\
$\left\|v_{h}\right\|_{L^{2}\left(q_{T}\right)}$ & 4.1796 & 4.6185 & 4.7589 & 4.7557 & 4.7291 \\
$\left\|L \varphi_{h}\right\|_{L^{2}\left(0, T ; H^{-1}(0,1)\right)}$ & 0.0391 & 0.0322 & 0.0162 & 0.0078 & 0.0037 \\
$\left\|v-v_{h}\right\|_{L^{2}\left(q_{T}\right)}$ & 2.4977 & 1.1341 & 0.5617 & 0.2418 & 0.1201 \\
$\left\|y-\lambda_{h}\right\|_{L^{2}\left(Q_{T}\right)}$ & $9.23 \times 10^{-2}$ & $4.56 \times 10^{-2}$ & $7.70 \times 10^{-3}$ & $1.71 \times 10^{-3}$ & $4.46 \times 10^{-4}$ \\
$\kappa$ & $6.12 \times 10^{8}$ & $1.44 \times 10^{10}$ & $1.51 \times 10^{11}$ & $1.55 \times 10^{12}$ & $1.54 \times 10^{13}$ \\
\hline
\end{tabular}

Table 4: Example EX1; $q_{T}=q_{2}^{2} ; r=10^{3}$.

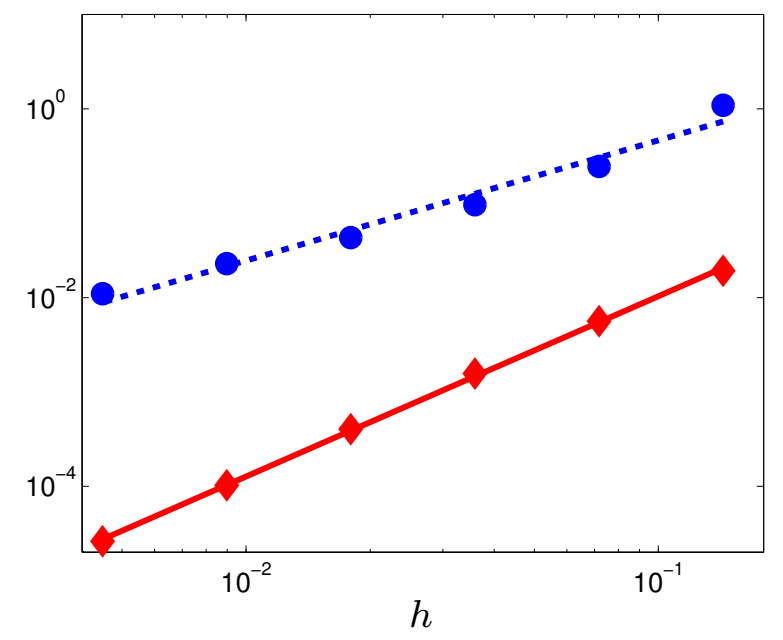

Figure 6: Example EX1; $r=10^{-1} ; q_{T}=q_{2.2}^{2} ;$ Norms $\left\|v-v_{h}\right\|_{L^{2}\left(q_{T}\right)}(\bullet)$ and $\left\|y-\lambda_{h}\right\|_{L^{2}\left(Q_{T}\right)}(\bullet)$ vs. $h$.
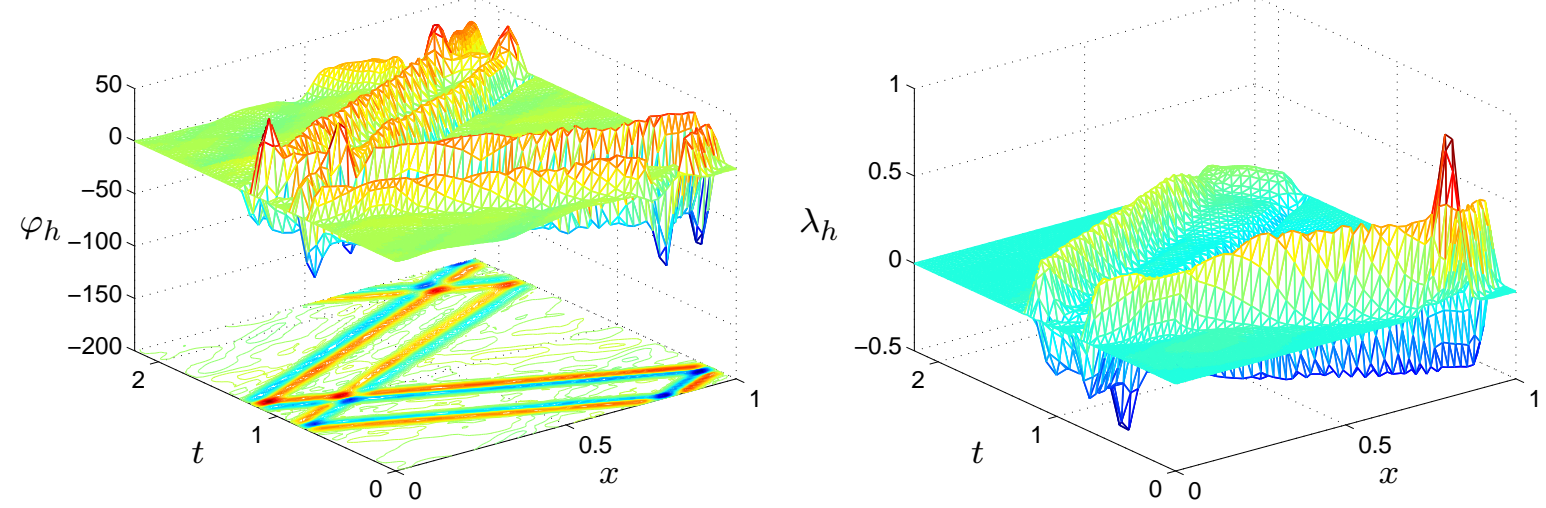

Figure 7: Example EX2; $r=10^{-1} ; q_{T}=q_{2.2}^{2}$ : Functions $\varphi_{h}$ (Left) and $\lambda_{h}$ (Right) over $Q_{T}$.

Similarly, Table 6 gives the value corresponding to the third example EX3, here with $\theta=1 / 2$. We obtain

$$
\left\|v-v_{h}\right\|_{L^{2}\left(q_{T}\right)} \approx e^{1.69} h^{0.53}, \quad\left\|L \varphi_{h}\right\|_{L^{2}\left(Q_{T}\right)} \approx e^{2.88} h^{0.56}, \quad\left\|y-\lambda_{h}\right\|_{L^{2}\left(Q_{T}\right)} \approx e^{-1.41} h^{1.32} .
$$

Table 7 gives the numerical results for the Example EX3 with $\theta=1 / 3$. We get

$$
\left\|v-v_{h}\right\|_{L^{2}\left(q_{T}\right)} \approx e^{1.54} h^{0.47}, \quad\left\|L \varphi_{h}\right\|_{L^{2}\left(Q_{T}\right)} \approx e^{2.91} h^{0.54}, \quad\left\|y-\lambda_{h}\right\|_{L^{2}\left(Q_{T}\right)} \approx e^{-1.52} h^{1.29} .
$$

Figure 8 displays the dual variable $\varphi_{h}$ and the primal variable $\lambda_{h}$ for $q_{T}=q_{2.2}^{2}$ and EX3 with $\theta=1 / 3$. The figures are again plotted with the mesh $\sharp 3$. 


\begin{tabular}{|c|ccccc|}
\hline$\sharp$ Mesh & 1 & 2 & 3 & 4 & 5 \\
\hline$h$ & $7.18 \times 10^{-2}$ & $3.59 \times 10^{-2}$ & $1.79 \times 10^{-2}$ & $8.97 \times 10^{-3}$ & $4.49 \times 10^{-3}$ \\
$\left\|v_{h}\right\|_{L^{2}\left(q_{T}\right)}$ & 4.8469 & 7.6514 & 10.9905 & 12.6256 & 12.9022 \\
$\|L \varphi\|_{L^{2}\left(0, T ; H^{-1}(0,1)\right)}$ & $3.13 \times 10^{1}$ & $2.91 \times 10^{1}$ & $1.82 \times 10^{1}$ & 6.8984 & 1.9257 \\
$\left\|v-v_{h}\right\|_{L^{2}\left(q_{T}\right)}$ & 8.4949 & 6.6975 & 3.2515 & $6.24 \times 10^{-1}$ & $5.31 \times 10^{-2}$ \\
$\left\|y-\lambda_{h}\right\|_{L^{2}\left(Q_{T}\right)}$ & $5.98 \times 10^{-2}$ & $2.78 \times 10^{-2}$ & $8.97 \times 10^{-3}$ & $2.01 \times 10^{-3}$ & $5.38 \times 10^{-4}$ \\
\hline
\end{tabular}

Table 5: Example EX2; $q_{T}=q_{2}^{2} ; r=10^{-1}$.

\begin{tabular}{|c|ccccc|}
\hline$\sharp$ Mesh & 1 & 2 & 3 & 4 & 5 \\
\hline$h$ & $7.18 \times 10^{-2}$ & $3.59 \times 10^{-2}$ & $1.79 \times 10^{-2}$ & $8.97 \times 10^{-3}$ & $4.49 \times 10^{-3}$ \\
$\left\|v_{h}\right\|_{L^{2}\left(q_{T}\right)}$ & 4.807 & 4.756 & 4.707 & 4.689 & 4.685 \\
$\left\|L \varphi_{h}\right\|_{L^{2}\left(0, T ; H^{-1}(0,1)\right)}$ & 3.858 & 2.965 & 1.881 & 1.232 & $8.61 \times 10^{-1}$ \\
$\left\|v-v_{h}\right\|_{L^{2}\left(q_{T}\right)}$ & 1.4382 & $8.73 \times 10^{-1}$ & $6.24 \times 10^{-1}$ & $4.24 \times 10^{-1}$ & $3.25 \times 10^{-1}$ \\
$\left\|y-\lambda_{h}\right\|_{L^{2}\left(Q_{T}\right)}$ & $6.86 \times 10^{-3}$ & $3.55 \times 10^{-3}$ & $1.19 \times 10^{-3}$ & $4.64 \times 10^{-4}$ & $1.96 \times 10^{-4}$ \\
\hline
\end{tabular}

Table 6: Example EX3 with $\theta=1 / 2 ; r=10^{-1} ; q_{T}=q_{2}^{2}$.
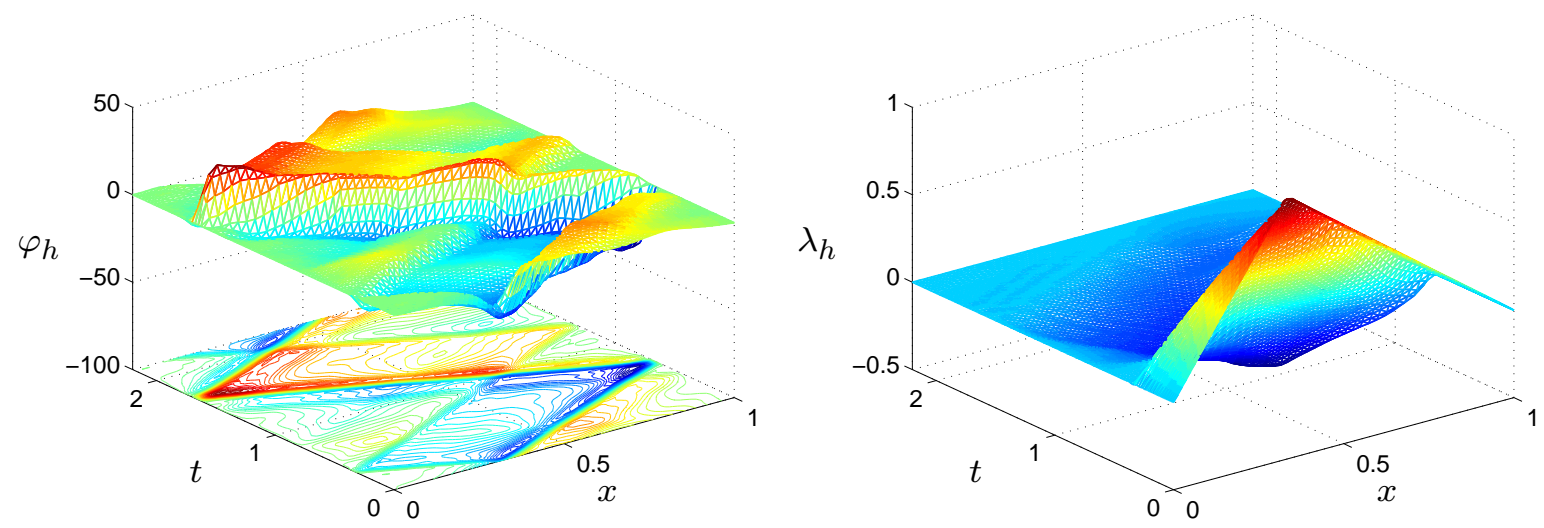

Figure 8: Example EX3 with $\theta=1 / 3 ; r=10^{-1} ; q_{T}=q_{2.2}^{2}$ : Functions $\varphi_{h}$ (Left) and $\lambda_{h}$ (Right).

\subsection{Comparison of $\|v\|_{L^{2}\left(q_{T}\right)}$ for various domains $q_{T}$ with the same mea- sure}

The optimization of the support domain $q_{T}$ is particularly relevant in the time dependent situation. As a first step in this direction, we compare numerically in this section the $L^{2}\left(q_{T}\right)$-norm of the control $v_{h}$ for various domain $q_{T}$ having the same measure. Along this section, we take $r=10^{-1}$ and $T=2.2$. The four domains we consider are $q_{T=2.2}^{i}$ for $i=0,1,2,3$ and are described in Section 4.1 .

Table 8 reports the $L^{2}$ norms of $v_{h}=-\varphi_{h} 1_{q_{T}^{i}}$ obtained with the finer mesh (mesh $\sharp 5$, see Table 1 ) associated to each domain.

Figure 9 displays the dual variable $\varphi_{h}$ and the primal one $\lambda_{h}$ associated to the initial data EX2 and control domains $q_{T}^{3}$.

Figure 10 displays the dual variable $\varphi_{h}$ and the primal one $\lambda_{h}$ associated to the initial data EX3, $\theta=1 / 3$ and control domains $q_{T}^{3}$.

We remark that any of these domains provides minimal norm controls for every initial data EX1-EX3. In fact, we suspect that the domains minimizing the $L^{2}$-norm of the control of minimal $L^{2}$-norm are strongly connected with the set generated by the characteristics of the initial data. This questions will be investigated in a future study. 


\begin{tabular}{|c|ccccc|}
\hline$\sharp$ Mesh & 1 & 2 & 3 & 4 & 5 \\
\hline$h$ & $7.18 \times 10^{-2}$ & $3.59 \times 10^{-2}$ & $1.79 \times 10^{-2}$ & $8.97 \times 10^{-3}$ & $4.49 \times 10^{-3}$ \\
$\left\|v_{h}\right\|_{L^{2}\left(q_{T}\right)}$ & 5.350 & 5.263 & 5.195 & 5.172 & 5.165 \\
$\left\|L \varphi_{h}\right\|_{L^{2}\left(0, T ; H^{-1}(0,1)\right)}$ & 4.230 & 3.339 & 2.095 & 1.382 & 1.022 \\
$\left\|v-v_{h}\right\|_{L^{2}\left(q_{T}\right)}$ & 1.3571 & $9.78 \times 10^{-1}$ & $6.91 \times 10^{-1}$ & $5.13 \times 10^{-1}$ & $3.69 \times 10^{-1}$ \\
$\left\|y-\lambda_{h}\right\|_{L^{2}\left(Q_{T}\right)}$ & $7.12 \times 10^{-3}$ & $3.23 \times 10^{-3}$ & $1.19 \times 10^{-3}$ & $4.82 \times 10^{-4}$ & $2.12 \times 10^{-4}$ \\
\hline
\end{tabular}

Table 7: Example EX3 with $\theta=1 / 3 ; r=10^{-1} ; q_{T}=q_{2}^{2}$.

\begin{tabular}{|c|cccc|}
\hline Initial data & $q_{T}^{0}$ & $q_{T}^{1}$ & $q_{T}^{2}$ & $q_{T}^{3}$ \\
\hline EX1 & 4.3677 & 3.8770 & 4.4808 & 5.5967 \\
EX2 & 11.9994 & 12.0973 & 10.6268 & 11.2624 \\
EX3, $\theta=1 / 3$ & 3.9946 & 4.5026 & 5.0132 & 5.0369 \\
\hline
\end{tabular}

Table 8: $L^{2}$-norm $\left\|v_{h}\right\|_{L^{2}\left(q_{T}\right)}$ for $q_{T}=q_{2.2}^{i}, i \in\{0,3\}$ for initial data EX1-EX3.
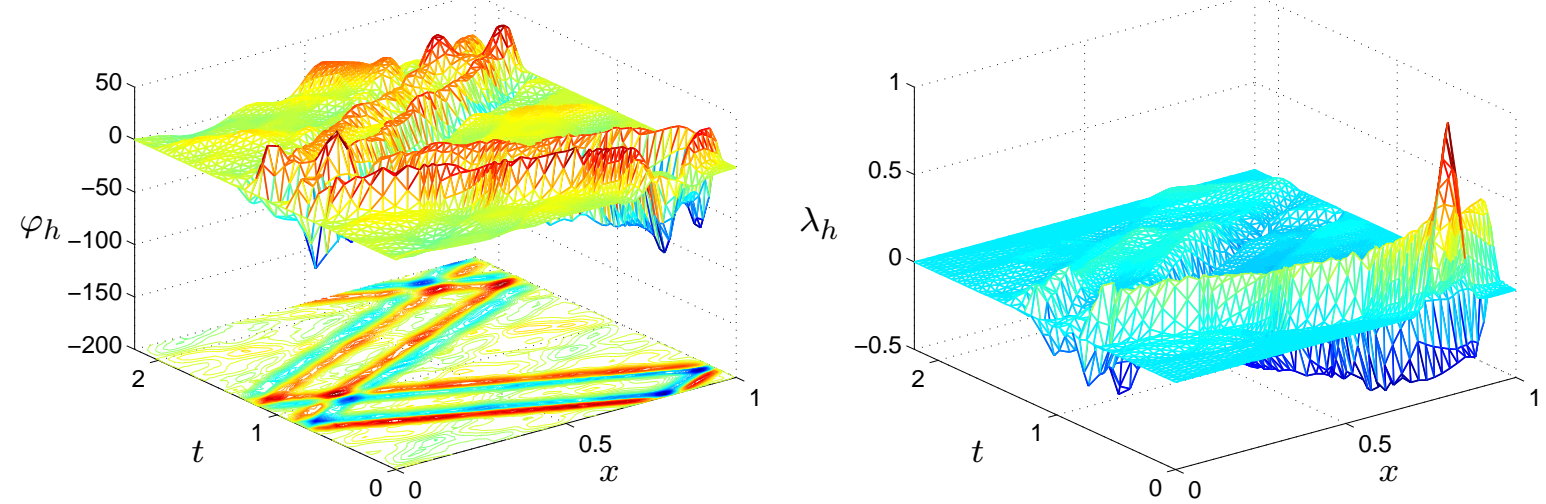

Figure 9: Example EX2: $q_{T}=q_{2.2}^{3}$ - Function $\varphi_{h}$ (Left) and $\lambda_{h}$ (Right) over $Q_{T}$.
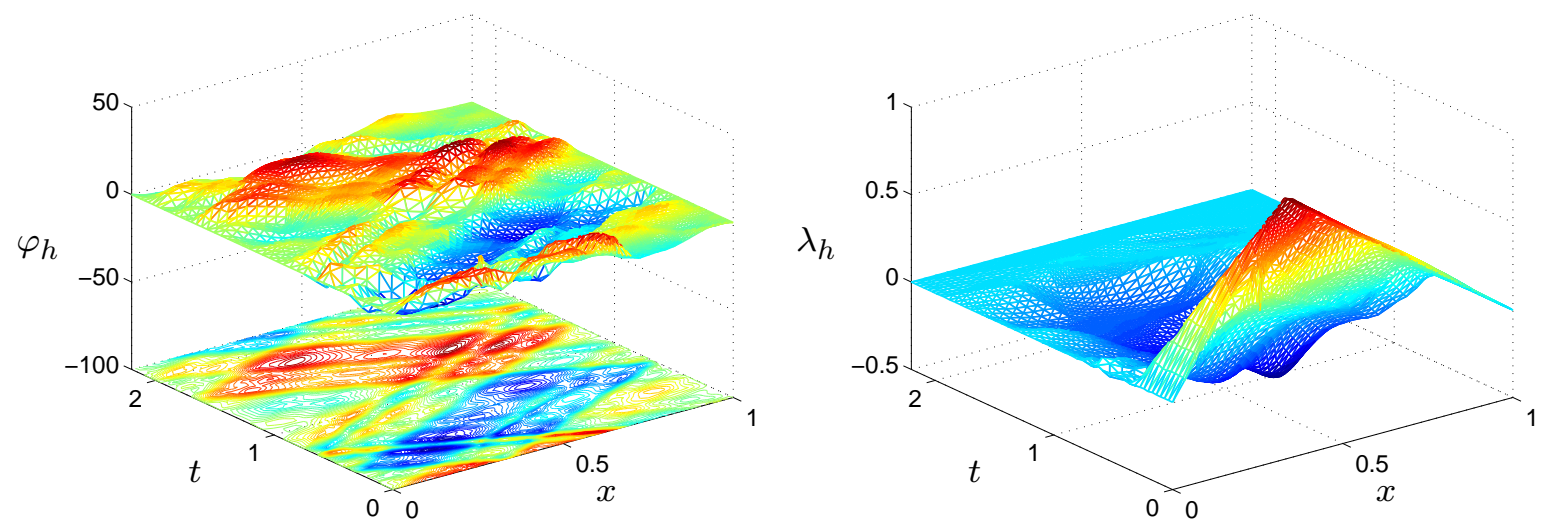

Figure 10: Example EX3, $\theta=1 / 3: q_{T}=q_{2.2}^{3}$ - Function $\varphi_{h}$ (Left) and $\lambda_{h}$ (Right) over $Q_{T}$.

\subsection{Behavior of the control as $\delta_{0} \searrow 0$}

The approach we have developed is valid for any support $q_{T}$ satisfying the hypothesis of Proposition 2.1. in particular arbitrarily thin domain. In this Section we study numerically the evolution of the norm of the controls of minimal $L^{2}$-norm supported in a time dependent domain $q_{T}$ when the measure of these domains goes to 0 . Precisely, we consider smaller and smaller values to the 
parameter $\delta_{0}$ defining the "thickness" of the domains $q_{T}$ as specified by (37)- (38).

In Table 9 we give the $L^{2}$ and $L^{2}\left(H^{-1}\right)$ norms of the controls obtained for the initial data EX1 and control domains $q_{T=2}^{2}$ for $\delta_{0}=\frac{10^{-1}}{2^{i}}$ for values of $i \in\{0,1,2, \ldots, 6\}$. As $\delta_{0} \searrow 0, q_{T}^{2}$ degenerates into the $C^{1}$-curve $\gamma_{T}^{2}$ defined by [35]; this curve satisfies the condition $\left|\left(\gamma_{T}^{2}\right)^{\prime}(t)\right|<1$ for all $t \in[0, T]$ assumed in [6] to prove the existence of $H^{-1}\left(\cup_{t \in(0, T)} \gamma(t) \times\{t\}\right)$ controls.

The numerical values suggest that both norms of the controls are not uniformly bounded (by above) with respect to $\delta_{0}$; this indicates that the $L^{2}$-controllability of (1) with control supported on the curve $\gamma_{T=2}^{2}$ (see 35 ) does not hold. Similar behaviors are obtained for the other domains considered in Section 4.1 when $\delta_{0} \searrow 0$. This does not contradict the result of [6] where the $H^{-1}\left(\cup_{t \in(0, T)} \gamma(t) \times\{t\}\right)$-controllability is proved in the limit situation.

\begin{tabular}{|c|ccccccc|}
\hline$\delta_{0}$ & $10^{-1}$ & $10^{-1} / 2$ & $10^{-1} / 2^{2}$ & $10^{-1} / 2^{3}$ & $10^{-1} / 2^{4}$ & $10^{-1} / 2^{5}$ & $10^{-1} / 2^{6}$ \\
\hline$\sharp$ triangles & 68740 & 68464 & 68402 & 68728 & 68422 & 68966 & 68368 \\
$\left\|v_{h}\right\|_{L^{2}\left(q_{T}\right)}$ & 4.830 & 7.330 & 11.574 & 18.805 & 29.735 & 47.315 & 123.970 \\
$\left\|v_{h}\right\|_{L^{2}\left(H^{-1}\right)}$ & 0.0035 & 0.0042 & 0.0066 & 0.0107 & 0.0170 & 0.0270 & 0.0704 \\
\hline
\end{tabular}

Table 9: Example EX1; $q_{T}=q_{2}^{2}$; Norms of the control $v_{h}$ obtained for the EX1 for control domains $q_{2}^{2}$ for different values of $\delta_{0}$.

\subsection{Other cases : Non constant velocity of propagation and $T<2$}

In order to illustrate our approach in a more challenging case we consider the wave equation with a non-constant velocity of propagation $c$ and control supported in a time dependent domain :

$$
\begin{cases}y_{t t}-\left(c(x) y_{x}\right)_{x}=v 1_{q_{T}}, & (x, t) \in Q_{T} \\ y(x, t)=0, & (x, t) \in \Sigma_{T} \\ y(x, 0)=y_{0}(x), \quad y_{t}(x, 0)=y_{1}(x), & x \in(0,1) .\end{cases}
$$

We take the velocity $c \in C^{\infty}(0,1)$ given by

$$
c(x)=\left\{\begin{array}{lll}
1, & & x \in[0,0.45] \\
\in[1,5], & \left(c^{\prime}(x)>0\right), & x \in(0.45,0.55) \\
5, & x \in[0.55,1] .
\end{array}\right.
$$

Note that the Fourier expansion developed in the appendix does not apply in this case. Although the inequality (11) is open in this more general case, we observe that the solution of the mixed formulation (24) still provides convergent approximations $\left\{v_{h}\right\}$ of controls. Figure 11 depicts the dual variable $\varphi_{h}$ and the primal variable $\lambda_{h}$ corresponding to the approximation of the control for problem (53), for initial data given by EX3 with $\theta=1 / 3$ and control domain $q_{2}^{2}$. The augmentation parameter is $r=10^{-1}$.

Since the control acts in a time dependent domain, the geometric controllability condition can hold for values of the controllability time $T$ which are smaller than 2 (we refer to [18). Figure 12 displays $\varphi_{h}$ and $\lambda_{h}$ corresponding to the exmaple EX3 for $\theta=1 / 3, T=1$ and $q_{T}=q_{1}^{2}$. We mention that in this section the domains $Q_{T}$ are discretized using uniform meshes formed by triangles of size $h \approx 10^{-2}$.

Another, even more challenging situation is the approximation of controls for problem 53 for shorter controllability times. In Figure 13 we display the results obtained for the initial data EX3, domain $q_{T=1}^{2}$ and the velocity of propagation is non constant in space and given by (54).

Analyzing the evolution of the norm of $\lambda_{h}$ with respect to the time, in all the three examples considered in these section it seems to have the controllability, although the hypotheses of Proposition 2.1 are not completely fulfilled. 

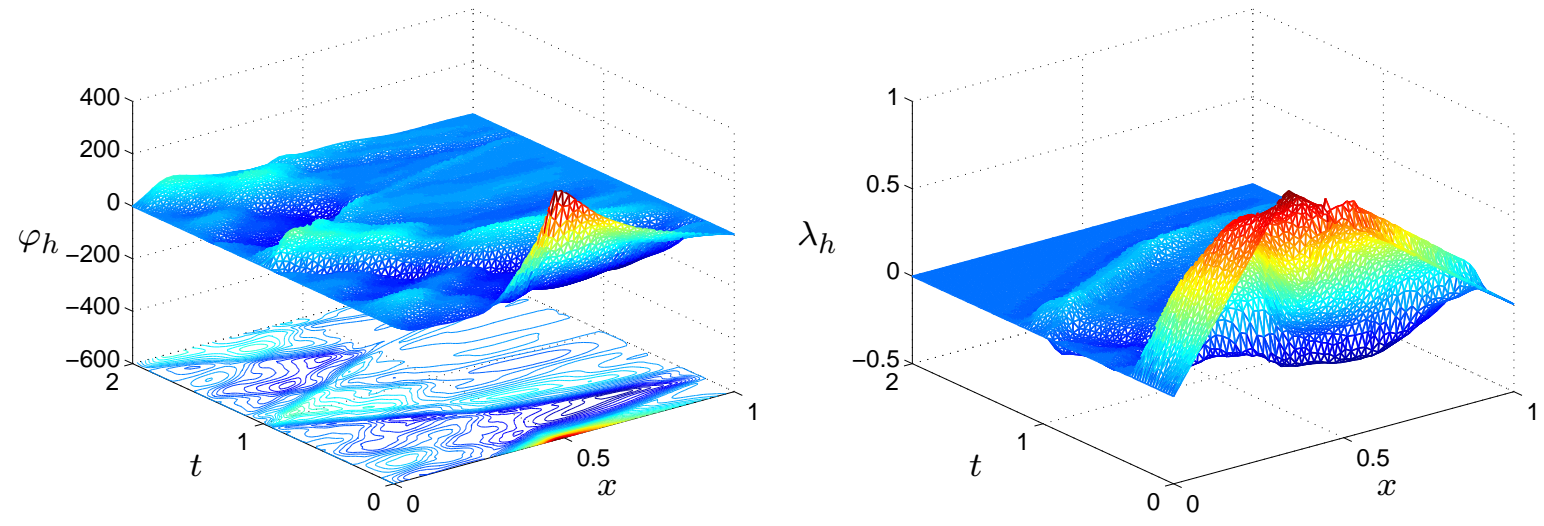

Figure 11: Example EX3, $\theta=1 / 3: q_{T}=q_{2}^{2}$ for a non-constant velocity of propagation - Function $\varphi_{h}$ (Left) and $\lambda_{h}$ (Right) over $Q_{T}$.
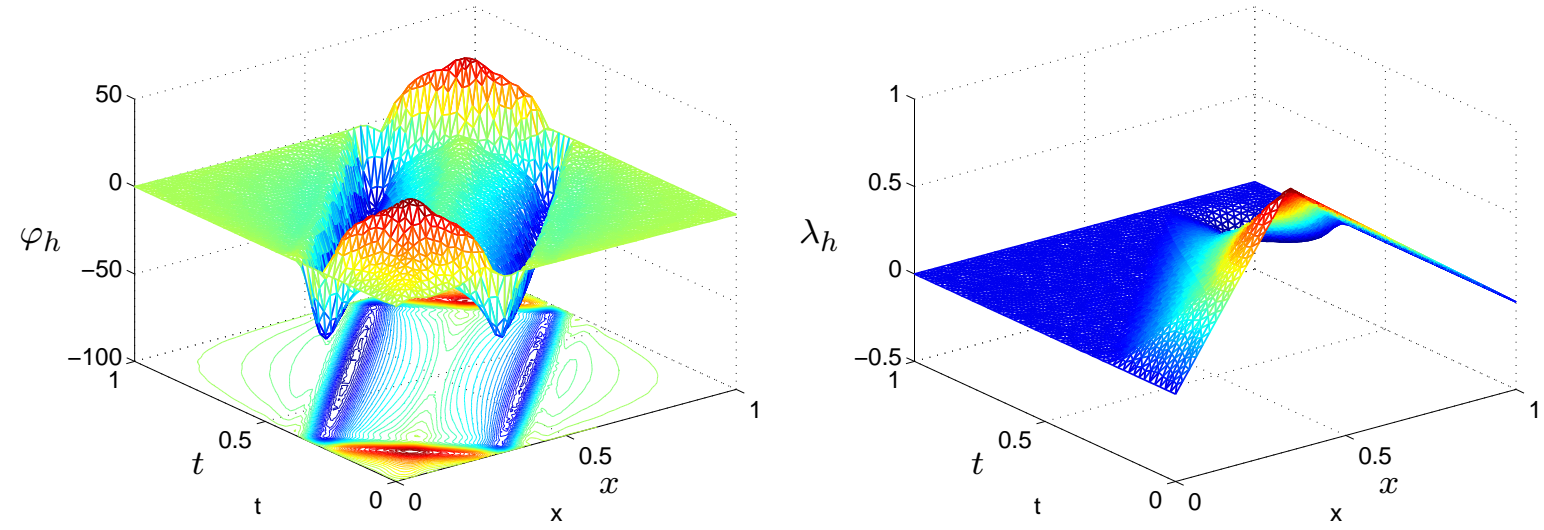

Figure 12: Example EX3, $\theta=1 / 3: q_{T}=q_{1}^{2}$ - Function $\varphi_{h}$ (Left) and $\lambda_{h}$ (Right) over $Q_{T}$.
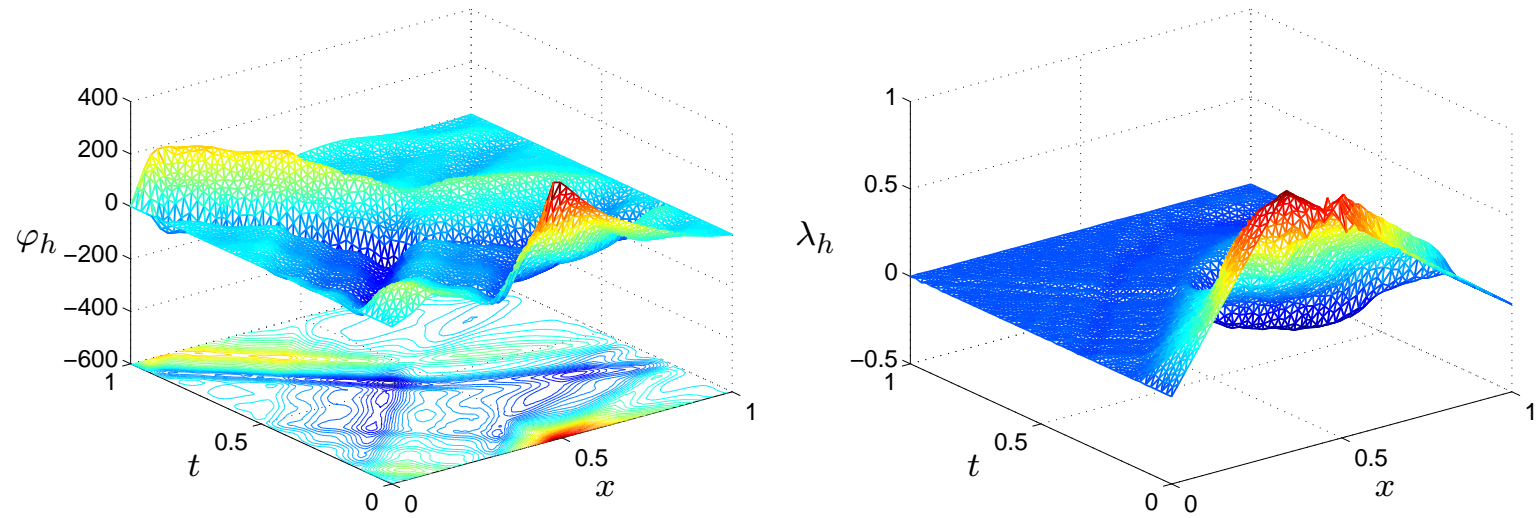

Figure 13: Example EX3, $\theta=1 / 3: q_{T}=q_{1}^{2}$ for a non-constant velocity of propagation - Function $\varphi_{h}$ (Left) and $\lambda_{h}$ (Right) over $Q_{T}$.

\subsection{Conjugate gradient for $J^{\star \star}$}

We illustrate here the Section 3.2 we minimize the functional $J^{\star \star}: L^{2}\left(Q_{T}\right) \rightarrow \mathbb{R}$ with respect to the variable $\lambda$. We recall that this minimization corresponds exactly to the resolution of the mixed formulation (24) by an iterative Uzawa type procedure. The conjugate gradient algorithm is given 
at the end of Section 3.2 In practice, each iteration amounts to solve a linear system involving the matrix $A_{r, h}$ of size $n_{h}=4 m_{h}$ (see $(49)$ ) which is sparse, symmetric and positive definite. We use the Cholesky method.

We consider the singular situation given by the example EX3 with $\theta=0.3, T=2$ and $q_{T}=q_{2}^{2}$. We take $\varepsilon=10^{-10}$ as a stopping threshold for the algorithm (that is the algorithm is stopped as soon as the norm of the residue $g^{n}$ at the iterate $n$ satisfies $\left\|g^{n}\right\|_{L^{2}\left(0, T ; H_{0}^{1}(0,1)\right)} \leq$ $\left.10^{-10}\left\|g^{0}\right\|_{L^{2}\left(0, T ; H_{0}^{1}(0,1)\right)}\right)$ or as the number of iterations is greater than 1000 . The algorithm is initiated with $\lambda^{0}=0$ in $Q_{T}$. Table 10 and 11 display the results for $r=10^{-1}$ and $r=10^{3}$.

We first check that this iterative method gives exactly the same approximation $\lambda_{h}$ than the previous direct method (where 49) is solved directly) since, from Proposition (3.1) problem 24] coincides with the minimization of $J^{\star \star}$ for $r>0$. Then, we observe that the number of iterates is sub-linear with respect to the dimension $m_{h}=\operatorname{card}\left(\left\{\lambda_{h}\right\}\right)$ of the approximated problem. Once again, this is in contrast with the behavior of the conjugate gradient algorithm when this latter is used to minimize $J^{\star}$ with respect to $\left(\varphi_{0}, \varphi_{1}\right)$ (see [22]).

Figure 14 displays the evolution of the residue $\left\|g^{n}\right\|_{L^{2}\left(0, T ; H_{0}^{1}(0,1)\right)} /\left\|g^{0}\right\|_{L^{2}\left(0, T ; H_{0}^{1}(0,1)\right)}$ with respect to the iteration $n$ for two values of the augmentation parameter : $r=10^{-1}$ and $r=10^{3}$. The computation has been done with the level mesh $\sharp 3$. As expected, we check that a larger value of $r$ improves significantly the convergence of the algorithm: recall that the gradient of $J^{\star \star}$ in $L^{2}\left(H^{1}\right)$ is given by: $\nabla J^{\star \star}(\lambda)=A_{r} \lambda-\Delta^{-1}\left(L \varphi_{0}\right):=-\Delta^{-1}(L \varphi)$ and that $r$ acts on the term $\|L \varphi\|_{L^{2}\left(H^{-1}\right)}$. For a fixe level of mesh, we observe however a lower error $\left\|\lambda_{h}-y\right\|_{L^{2}\left(Q_{T}\right)}$ for $r=10^{-1}$.

\begin{tabular}{|c|ccccc|}
\hline$\sharp$ Mesh & 1 & 2 & 3 & 4 & 5 \\
\hline$h$ & $7.18 \times 10^{-2}$ & $3.59 \times 10^{-2}$ & $1.79 \times 10^{-2}$ & $8.97 \times 10^{-3}$ & $4.49 \times 10^{-3}$ \\
$\sharp$ iterate & 307 & 414 & 624 & 967 & 1000 \\
$\left\|\lambda_{h}-y\right\|_{L^{2}\left(Q_{T}\right)}$ & $1.28 \times 10^{-2}$ & $4.77 \times 10^{-3}$ & $1.5 \times 10^{-3}$ & $6.2 \times 10^{-4}$ & $3.52 \times 10^{-6}$ \\
\hline
\end{tabular}

Table 10: Conjugate gradient algorithm. EX3 with $\theta=1 / 3$, for control domain $q_{2}^{2}$ and $r=10^{-1}$.

\begin{tabular}{|c|ccccc|}
\hline$\sharp$ Mesh & 1 & 2 & 3 & 4 & 5 \\
\hline$h$ & $7.18 \times 10^{-2}$ & $3.59 \times 10^{-2}$ & $1.79 \times 10^{-2}$ & $8.97 \times 10^{-3}$ & $4.49 \times 10^{-3}$ \\
$\sharp$ iterate & 87 & 105 & 119 & 140 & 166 \\
$\left\|\lambda_{h}-y\right\|_{L^{2}\left(Q_{T}\right)}$ & $1.15 \times 10^{-1}$ & $5.2 \times 10^{-2}$ & $1.65 \times 10^{-2}$ & $6.03 \times 10^{-3}$ & $2.89 \times 10^{-3}$ \\
\hline
\end{tabular}

Table 11: Conjugate gradient algorithm. EX3 with $\theta=1 / 3$, for control domain $q_{2}^{2}$ and $r=10^{3}$.

\section{Concluding remarks and perspectives}

We have extended in this work the contribution [10 to a non-cylindrical situation where the support of the controls depend on the time variable. The numerical approximation is based on a direct resolution of the controllability problem through a mixed formulation involving the dual adjoint variable and a Lagrange multiplier, which turns out to coincide with the primal state of the wave equation to be controlled. The well-posedness of this mixed formulation is the consequence of a generalized observability inequality deduced from [6] (and equivalent to the controllability of the equation). The approach leads to a variational formulation over time-space functional Hilbert space without distinction between the time and the space variable and is very appropriate to non-cylindrical situations.

At the practical level, the discrete mixed time-space formulation is solved in a systematic way in the framework of the finite element theory: in contrast to the classical approach, there is no need 


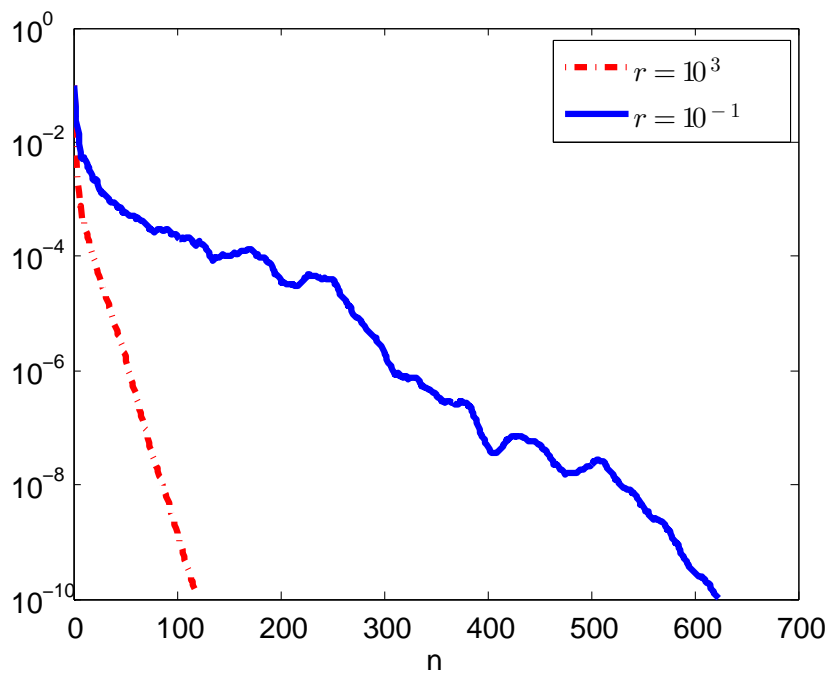

Figure 14: Example EX3. Evolution of the residue $\left\|g^{n}\right\|_{L^{2}\left(0, T ; H_{0}^{1}(0,1)\right)} /\left\|g^{0}\right\|_{L^{2}\left(0, T ; H_{0}^{1}(0,1)\right)}$ w.r.t. the iterate $n$.

to take care of the time discretization nor of the stability of the resulting scheme, which is often a delicate issue. The resolution amounts to solve a sparse symmetric linear system. As discussed in [10, Section 4.3 (but not employed here), the space-time discretization of the domain allows an adaptation of the mesh so as to reduce the computational cost and capture the main features of the solution.

The numerical experiments reported in this work suggest a very good behavior of the approach: the strong convergence of the sequences $\left\{v_{h}\right\}_{h>0}$, approximation of the controls of minimal square integrable norm, are clearly observed as the discretization parameter $h$ tends to zero (as the consequence of the uniform inf-sup discrete property).

As briefly discussed in Section 4.7, this work opens now the possibility to optimize the control $v$ of minimal $L^{2}\left(q_{T}\right)$-norm with respect the support $q_{T}$ (equivalently in our case, with respect to the curves $a$ and $b$, see (6) $)$ in the spirit of [23, 24, 25]: for any $\left(y_{0}, y_{1}\right) \in \boldsymbol{H}, T>0$ and $L \in(0,1)$, the problem reads :

$$
\inf _{q_{T} \in C_{L}}\left\|v_{q_{T}}\right\|_{L^{2}\left(q_{T}\right)}, \quad C_{L}=\left\{q_{T}: q_{T} \subset Q_{T},\left|q_{T}\right|=L\left|Q_{T}\right| \text { and such that } 11 \text { holds }\right\}
$$

where $v_{q_{T}}$ denotes the control of minimal $L^{2}\left(q_{T}\right)$ norm for (1) distributed over $q_{T}$.

Eventually, we also mention that this approach which consists in solving directly the optimality conditions of a controllability problem may be employed to solve inverse problems where, for instance, the solution of the wave equation has to be recovered from a partial observation, typically localized on a sub-domain $q_{T}$ of the working domain: actually, the optimality conditions associated to a least-square type functional can be expressed as a mixed formulation very closed to 24 . These last two issues will be analyzed in a future work.

\section{A Appendix: Fourier expansion of the control of minimal $L^{2}\left(q_{T}\right)$-norm}

We expand in this appendix in term of Fourier series the control of minimal $L^{2}\left(q_{T}\right)$-norm $v$ for (1) and the corresponding controlled solution $y$. We use these expansions in Section 4.6 to evaluate with respect to $h$, the error $\left\|y-\lambda_{h}\right\|_{L^{2}\left(Q_{T}\right)}$ and $\left\|v-v_{h}\right\|_{L^{2}\left(q_{T}\right)}$ where the sequence $\left(\varphi_{h}, \lambda_{h}\right)_{h>0}$ 
solves the discrete mixed formulation (39). We use the characterization of the couple $(y, v)$ in term of the adjoint solution $\varphi$ (see 5), unique minimizer in $\boldsymbol{H}$ of $J^{\star}$ defined by (8).

We first note $\left(a_{p}, b_{p}\right)_{(p>0)}$ the Fourier coefficients in $l^{2}(\mathbb{N}) \times h^{-1}(\mathbb{N})$ of the minimizer $\left(\varphi_{0}, \varphi_{1}\right) \in$ $L^{2}(0,1) \times H^{-1}(0,1)$ of $(8)$, such that $\left(\varphi_{0}(x), \varphi_{1}(x)\right)=\sum_{p>0}\left(a_{p}, b_{p}\right) \sin (p \pi x)$. The adjoint state takes the form

$$
\varphi(x, t)=\sum_{p>0}\left(a_{p} \cos (p \pi t)+\frac{b_{p}}{p \pi} \sin (p \pi t)\right) \sin (p \pi x) .
$$

We get

$$
\begin{aligned}
\iint_{q_{T}}|\varphi|^{2} d x d t & =\sum_{p, q>0} a_{p} \overline{a_{q}} \iint_{q_{T}} \cos (p \pi t) \cos (q \pi t) \sin (p \pi x) \sin (q \pi x) d x d t \\
& +\sum_{p, q>0} a_{p} \overline{b_{q}} \iint_{q_{T}} \cos (p \pi t) \frac{\sin (q \pi t)}{q \pi} \sin (p \pi x) \sin (q \pi x) d x d t \\
& +\sum_{p, q>0} b_{p} \overline{a_{q}} \iint_{q_{T}} \frac{\sin (p \pi t)}{p \pi} \cos (q \pi t) \sin (p \pi x) \sin (q \pi x) d x d t \\
& +\sum_{p, q>0} b_{p} \overline{b_{q}} \iint_{q_{T}} \frac{\sin (p \pi t)}{p \pi} \frac{\sin (q \pi t)}{q \pi} \sin (p \pi x) \sin (q \pi x) d x d t
\end{aligned}
$$

and

$$
\left\{\begin{array}{l}
\int_{0}^{1} \varphi_{0}(x) y_{1}(x) d x=\sum_{p>0} a_{p} \int_{0}^{1} y_{1}(x) \sin (p \pi x) d x \\
<\varphi_{1}, y_{0}>_{H^{-1}, H_{0}^{1}}=\sum_{p>0} b_{p} \int_{0}^{1} y_{0, x}(x) \frac{\cos (p \pi x)}{p \pi} d x .
\end{array}\right.
$$

The optimality equation associated to the functional $J^{\star}$ (see 8) then reads

$$
D J^{\star}\left(\varphi_{0}, \varphi_{1}\right) \cdot\left(\overline{\varphi_{0}}, \overline{\varphi_{1}}\right)=\iint_{q_{T}} \varphi \bar{\varphi} d x d t+<\overline{\varphi_{1}}, y_{0}>_{H^{-1}, H_{0}^{1}}-\int_{0}^{1} \overline{\varphi_{0}} y_{1} d x=0, \quad \forall\left(\overline{\varphi_{0}}, \overline{\varphi_{1}}\right) \in \boldsymbol{H}
$$

and can be rewritten in term of the $\left(a_{p}, b_{p}\right)_{p>0}$ as follows :

$$
<\left(\begin{array}{c}
\left\{\overline{a_{p}}\right\}_{p>0} \\
\left\{\overline{b_{p}}\right\}_{p>0}
\end{array}\right), \mathcal{M}_{q_{T}}\left(\begin{array}{c}
\left\{a_{p}\right\}_{p>0} \\
\left\{b_{p}\right\}_{p>0}
\end{array}\right)>=<\left(\begin{array}{c}
\left\{\overline{a_{p}}\right\}_{p>0} \\
\left\{\overline{b_{p}}\right\}_{p>0}
\end{array}\right), \mathcal{F}_{y_{0}, y_{1}}>\quad \forall\left(\overline{a_{p}}, \overline{b_{p}}\right) \in l^{2}(\mathbb{N}) \times h^{-1}(\mathbb{N})
$$

where $\mathcal{M}_{q_{T}}$ denotes a symmetric positive definite matrix and $\mathcal{F}_{y_{0}, y_{1}}$ a vector obtained from (55) and (56) respectively. The resolution of the infinite dimensional system (57) (reduced to a finite dimension one by truncation of the sums) allows an approximation of the minimizer $\left(\varphi_{0}, \varphi_{1}\right)$ of $J^{\star}$ (see (8)), and then of $\varphi$, solution both of the boundary value problem (5) and of the mixed formulation (24).

Finally, we use that the corresponding control is given by $v=-\varphi 1_{q_{T}}$. We expand the corresponding controlled solution as $y(x, t)=\sum_{p>0} b_{p}(t) \sin (p \pi x)$ where, for all $p>0$, the function $b_{p}$ solves the ODE

$$
\left\{\begin{array}{l}
b_{p}^{\prime \prime}(t)+(p \pi)^{2} b_{p}(t)=-\sum_{q>0}\left(a_{q} \cos (q \pi t)+b_{q} \sin (q \pi t)\right) c_{p, q}(t):=f_{p}(t), \quad t \in(0, T), \\
\sum_{p>0} b_{p}(0) \sin (p \pi x)=y_{0}(x), \quad \sum_{p>0} b_{p}^{\prime}(0) \sin (p \pi x)=y_{1}(x)
\end{array}\right.
$$

with $c_{p, q}(t):=2 \int_{a(t)}^{b(t)} \sin (p \pi x) \sin (q \pi x) d x$. The function $b_{p}$ is given by

$$
\begin{aligned}
b_{p}(t):= & C_{1 p} \cos (p \pi t)+\frac{C_{2 p}}{p \pi} \sin (p \pi t) \\
& +\frac{1}{p \pi}\left(\sin (p \pi t) \int_{0}^{t} \cos (p \pi s) f_{p}(s) d s-\cos (p \pi t) \int_{0}^{t} \sin (p \pi s) f_{p}(s) d s\right)
\end{aligned}
$$


where $\left(C_{1 p}, C_{2 p}\right)_{p>0}$ are the Fourier's coefficients of the initial data $\left(y_{0}, y_{1}\right) \in \boldsymbol{V}$.

\section{References}

[1] C. Bardos, G. Lebeau, and J. Rauch, Sharp sufficient conditions for the observation, control, and stabilization of waves from the boundary, SIAM J. Control Optim., 30 (1992), pp. 1024-1065.

[2] M. Bernadou And K. Hassan, Basis functions for general Hsieh-Clough-Tocher triangles, complete or reduced, Internat. J. Numer. Methods Engrg., 17 (1981), pp. 784-789.

[3] P. B. Bochev and M. D. Gunzburger, Least-squares finite element methods, vol. 166 of Applied Mathematical Sciences, Springer, New York, 2009.

[4] J. H. Bramble and J. E. Pasciak, Least-squares methods for Stokes equations based on a discrete minus one inner product, J. Comput. Appl. Math., 74 (1996), pp. 155-173. TICAM Symposium (Austin, TX, 1995).

[5] F. Brezzi And M. Fortin, Mixed and hybrid finite element methods, vol. 15 of Springer Series in Computational Mathematics, Springer-Verlag, New York, 1991.

[6] C. Castro, Exact controllability of the 1-D wave equation from a moving interior point, ESAIM Control Optim. Calc. Var., 19 (2013), pp. 301-316.

[7] D. Chapelle and K.-J. Bathe, The inf-sup test, Comput. \& Structures, 47 (1993), pp. 537545 .

[8] P. G. Ciarlet, The finite element method for elliptic problems, vol. 40 of Classics in Applied Mathematics, Society for Industrial and Applied Mathematics (SIAM), Philadelphia, PA, 2002. Reprint of the 1978 original [North-Holland, Amsterdam; MR0520174 (58 \#25001)].

[9] N. Cîndea, E. Fernández-Cara, And A. Münch, Numerical controllability of the wave equation through primal methods and carleman estimates, ESAIM Control Optim. Calc. Var., 19 (2013), pp. 1076-1108.

[10] N. Cîndea ANd A. MÜNCH, A mixed formulation for the direct approximation of the control of minimal $L^{2}$-norm for linear type wave equations, http://hal.archives-ouvertes.fr/hal-00853767.

[11] L. Cui, X. Liu, And H. GaO, Exact controllability for a one-dimensional wave equation in non-cylindrical domains, J. Math. Anal. Appl., 402 (2013), pp. 612-625.

[12] J. W. DANiel, The approximate minimization of functionals, Prentice-Hall Inc., Englewood Cliffs, N.J., 1971.

[13] D. A. Dunavant, High degree efficient symmetrical Gaussian quadrature rules for the triangle, Internat. J. Numer. Methods Engrg., 21 (1985), pp. 1129-1148.

[14] M. Fortin And R. Glowinski, Augmented Lagrangian methods, vol. 15 of Studies in Mathematics and its Applications, North-Holland Publishing Co., Amsterdam, 1983. Applications to the numerical solution of boundary value problems, Translated from the French by B. Hunt and D. C. Spicer.

[15] R. Glowinski, Handbook of numerical analysis. Vol. IX, Handbook of Numerical Analysis, IX, North-Holland, Amsterdam, 2003. Numerical methods for fluids. Part 3. 
[16] R. Glowinski and J.-L. Lions, Exact and approximate controllability for distributed parameter systems, in Acta numerica, 1995, Acta Numer., Cambridge Univ. Press, Cambridge, 1995, pp. 159-333.

[17] R. Glowinski, J.-L. Lions, And J. He, Exact and approximate controllability for distributed parameter systems, vol. 117 of Encyclopedia of Mathematics and its Applications, Cambridge University Press, Cambridge, 2008. A numerical approach.

[18] A. Y. Khapalov, Controllability of the wave equation with moving point control, Appl. Math. Optim., 31 (1995), pp. 155-175.

[19] J.-L. Lions, Contrôlabilité exacte, perturbations et stabilisation de systèmes distribués. Tome 1, vol. 8 of Recherches en Mathématiques Appliquées [Research in Applied Mathematics], Masson, Paris, 1988. Contrôlabilité exacte. [Exact controllability], With appendices by E. Zuazua, C. Bardos, G. Lebeau and J. Rauch.

[20] P. Martin, L. Rosier, And P. Rouchon, Null controllability of the structurally damped wave equation with moving control, SIAM J. Control Optim., 51 (2013), pp. 660-684.

[21] A. Meyer, A simplified calculation of reduced hct-basis functions in a finite element context, Comput. Methods Appl. Math., 12 (2012), pp. 486-499.

[22] A. MüNCH, A uniformly controllable and implicit scheme for the 1-D wave equation, M2AN Math. Model. Numer. Anal., 39 (2005), pp. 377-418.

[23] — Optimal design of the support of the control for the 2-D wave equation: a numerical method, Int. J. Numer. Anal. Model., 5 (2008), pp. 331-351.

[24] A. MÜnch and F. Periago, Optimal distribution of the internal null control for the onedimensional heat equation, J. Differential Equations, 250 (2011), pp. 95-111.

[25] F. Periago, Optimal shape and position of the support for the internal exact control of a string, Systems Control Lett., 58 (2009), pp. 136-140.

[26] P.-F. YAO, On the observability inequalities for exact controllability of wave equations with variable coefficients, SIAM J. Control Optim., 37 (1999), pp. 1568-1599. 